\title{
TeV Astronomy
}

\author{
Frank M. RIEGER ${ }^{1,4}$, Emma de OÑA-WILHELMI $^{1,2}$, Felix A. AHARONIAN $^{1,3}$ \\ ${ }^{1}$ Max-Planck-Institut für Kernphysik, P.O. Box 103980, 69029 Heidelberg, Germany \\ ${ }^{2}$ Institut de Ciencies de L'Espai (IEEC-CSIC), Campus UAB, Torre C5, 08193 Bellaterra, Spain \\ ${ }^{3}$ Dublin Institute for Advanced Studies, 31 Fitzwilliam Place, Dublin 2, Ireland \\ ${ }^{4}$ European Associated Laboratory for Gamma-Ray Astronomy, jointly supported by CNRS and MPG \\ E-mail: †frank.rieger@mpi-hd.mpg.de,wilhelmi@aliga.ieec.uab.es, felix.aharonian@mpi-hd.mpg.de \\ Received 2012; accepted 2013
}

\begin{abstract}
With the successful realization of the current-generation of ground-based detectors, TeV Astronomy has entered into a new era. We review recent advances in VHE astronomy, focusing on the potential of Imaging Atmospheric Cherenkov Telescopes (IACTs), and highlight astrophysical implications of the results obtained within recent years.
\end{abstract}

Keywords TeV Astronomy, Gamma-Rays, Cherenkov Telescopes, High-Energy Astrophysics

PACS numbers 03.67.Lx, 03.65.Yz, 82.56.Jn

Contents

$1 \mathrm{TeV}$ Astronomy

1.1 Introduction

1.2 Ground-based Detection Technique

1.3 Future IACT Arrays

$2 \mathrm{TeV}$ Sources

2.1 Supernova Remnants

2.2 Pulsars

2.3 Pulsar Wind Nebulae

$2.4 \mathrm{TeV}$ Binary Systems

2.5 Galactic Centre

2.6 Blazars

2.7 Radio Galaxies

2.8 Starburst Galaxies

2.9 Candidates (GRBs, Clusters, Passive BHs) 20

3 Physics Impact of Recent Results

3.1 CR and Galactic Gamma-Ray Sources

3.2 Relativistic Outflows and AGNs

4 Conclusions and Perspectives

Acknowledgements

References

\section{TeV Astronomy}

\subsection{Introduction}

The discovery of more than 100 extraterrestrial sources of Very High Energy (VHE, $\geqslant 100 \mathrm{GeV}$ ) or TeV gammaradiation belongs to the most remarkable achievements of the last decade in astrophysics. The strong impact of these discoveries on several topical areas of modern astrophysics and cosmology are recognised and highly appreciated by different astronomical communities. The implications of the results obtained with ground-based $\mathrm{TeV}$ gamma-ray detectors are vast; they extend from the origin of cosmic rays to the origin of Dark Matter, from processes of acceleration of particles by strong shock waves to the magnetohydrodynamics of relativistic outflows, from distribution of atomic and molecular gas in the Interstellar Medium to the intergalactic radiation and magnetic fields.

$\mathrm{TeV}$ gamma-rays are copiously produced in environments where effective acceleration of particles (electrons, protons, and nuclei) is accompanied by their intensive interactions with the surrounding gas and radiation fields. These interactions contribute significantly to the bolometric luminosities of young Supernova Remnants (SNRs), Star Forming Regions (SFRs), Giant Molecular Clouds (GMCs), Pulsar Wind Nebulae (PWNe), compact Binary Systems, Active Galactic Nuclei (AGNs) and Radio Galaxies (RGs), etc..

The fast emergence of gamma-ray astronomy from an underdeveloped branch of cosmic-ray studies to a truly astronomical discipline is explained by the successful realization of the great potential of stereoscopic arrays of Imaging Atmospheric Cherenkov Telescopes (IACTs) which act as effective multifunctional tools for deep studies of cosmic gamma-radiation.

Being recognised as one of the most informative windows to the non-thermal Universe, the VHE domain of gamma-rays provides also means for probing fundamen- 
tal physics beyond the reach of terrestrial accelerators. In particular, the indirect search for Dark Matter and tests of quantum gravity using these energetic gammarays are amongst the high priority objectives of the current and future projects with involvement of groundbased gamma-ray detectors. In this regard, the $\mathrm{TeV}$ gamma-ray astronomy is considered a key component of the new interdisciplinary research area called AstroParticle Physics.

\subsection{Ground-based Detection Technique}

The atmosphere of the Earth is not transparent to gamma-rays, therefore their direct registration requires platforms in space. The currently operating Fermi Large Area Telescope (Fermi-LAT; formerly GLAST) is a powerful satellite-borne instrument designed for deep surveys with a very large field view of the order of 2 steradian. Presently, the study of the sky in $\mathrm{MeV}$ and $\mathrm{GeV}$ gamma-rays by Fermi-LAT is complemented by a somewhat smaller-scale telescope on the italian X-ray and gamma-ray satellite AGILE (Astro-rivelatore Gamma a Immagini LEggero). The angular resolution of these instruments below $1 \mathrm{GeV}$ is quite modest (larger than $1^{\circ}$ ), but it becomes significantly better at higher energies, approaching $0.1^{\circ}$ above $10 \mathrm{GeV}$. Fermi-LAT covers a very broad energy region of primary gamma-rays extending from tens of $\mathrm{MeV}$ to hundreds of $\mathrm{GeV}$ (HE; up to 300 $\mathrm{GeV}$ ). However, beyond $10 \mathrm{GeV}$ the gamma-ray fluxes are generally very faint, so that the effective detection area of Fermi-LAT cannot provide adequate statistics for comprehensive spectral and temporal studies in the VHE domain.

There is not much hope that space platforms could offer, in any time in the foreseeable future, instruments with detection areas significantly exceeding $1 \mathrm{~m}^{2}$. This dramatically reduces the potential of studies of VHE gamma-rays from space. Fortunately, at these energies an alternative method can be exploited based on the registration of atmospheric showers, either directly or through their Cherenkov radiation. The faint and brief Cherenkov signal of relativistic electrons produced during the development of the electromagnetic cascades in the atmosphere, lasts only several nanoseconds, but it is sufficient for detection using large optical reflectors equipped with fast optical receivers. With a telescope consisting of a $10 \mathrm{~m}$ diameter reflector and a multichannel camera of pixel size of $\sim 1 / 4$ degree and a fieldof-view of $\sim 3$ degree, primary gamma-rays of energy $E \geqslant 100 \mathrm{GeV}$ can be effectively collected across groundlevel distances as large as $100 \mathrm{~m}$ providing a huge area for the detection of primary gamma-rays, $A_{\text {eff }} \geqslant 10^{4} \mathrm{~m}^{2}$. The total number of photons in the registered Cherenkov light image is proportional to the primary (absorbed in the atmosphere) energy, the orientation of the image cor- relates with the arrival direction of the gamma-ray photon, and the shape of the image contains information about the origin of the primary particle (a proton or a photon?). The stereoscopic observations of air showers with two or more telescopes located at distances of about $100 \mathrm{~m}$ from each other, provide effective rejection of hadronic showers (by a factor of 100), as well as good angular resolution (better than $0.1^{\circ}$ ) and energy resolution (better than 15 per cent). At energies around 1 $\mathrm{TeV}$, this results in a minimum detectable energy flux as low as $3 \times 10^{-13} \mathrm{erg} / \mathrm{cm}^{2} \mathrm{~s}$ (see e.g. [1]) This is much better than in any other gamma-ray domain, including the $\mathrm{GeV}$ energy band, where the sensitivity of Fermi LAT cannot compete with the performance already achieved by the H.E.S.S., MAGIC and VERITAS IACT arrays in the TeV energy band. Thanks to the very large collection area, the IACT technique provides large gammaray photon statistics even from relatively modest $\mathrm{TeV}$ gamma-ray emitters. In combination with good energy and angular resolutions, the gamma-ray photon statistics appears to be adequate for deep morphological, spectroscopic and temporal studies. This also makes the IACT arrays powerful multifunctional and multi-purpose tools for the exploration of a broad range of non-thermal objects and phenomena. The potential of the IACT arrays has been convincingly demonstrated by the H.E.S.S., MAGIC and VERITAS collaborations (see, e.g. [2], and references therein).

The IACT arrays are capable to study not only pointlike, but also extended sources with an angular size up to 1 degree or somewhat larger. Moreover, the high flux sensitivity and relatively large $\left(\geqslant 4^{\circ}\right)$ field of view of IACT arrays allow rather effective all-sky surveys as demonstrated by the H.E.S.S. collaboration. On the other hand, the potential of IACT arrays is rather limited for the search of very extended structures like the galactic plane diffuse emission or the huge radio lobes of the nearby radio galaxy Centaurus A. IACT arrays have a limited capability for "hunting" of solitary events like the possible VHE counterparts of Gamma Ray Bursts. In this regard, the detection technique based on direct registration of particles (leptons, hadrons and photons) of extensive air showers (EAS) is a complementary approach to the IACT technique.

The traditional EAS technique, based on scintillators or water Cherenkov detectors spread over large areas, was originally designed for the detection of cosmic rays at $\mathrm{PeV}$ and $\mathrm{EeV}$ energies. In order to adopt this technique to gamma-ray astronomy, the energy threshold needs to be reduced by two or three orders of magnitude. This can be achieved using dense particle arrays located on very high altitudes. The feasibility of both approaches recently have been successfully demonstrated by the ARGO and Milagro collaborations. In particular, several very extended sources have been reported by the 
Milagro group. These results, as well as the potential for continuous monitoring of a significant part of the sky, which might lead to exciting discoveries of yet unknown VHE transient phenomena in the Universe, strongly support the proposals of constructing high altitude EAS detectors (see [1] for a review) like HAWC, a High Altitude Water Cherenkov Experiment, presently under construction on a site close to Sierra Negra, Mexico [3]. The 5 yr-survey sensitivity of HAWC above $1 \mathrm{TeV}$ is expected to be comparable to the sensitivity of Fermi-LAT at 1 GeV. Thus HAWC will be complementary to Fermi for continuous monitoring of more than 1 steradian fraction of the sky at $\mathrm{TeV}$ energies. At higher energies, recently a new project called LHAASO (Large High Altitude Air Shower Observatory) has been suggested. The proposed huge detector facility at Yangbajing, China, will consist of several sub-arrays for the detection of the electromagnetic and muon components of air showers. They will cover a huge area, and can achieve an impressive sensitivity at energies of several tens of TeV (see Fig. 1).

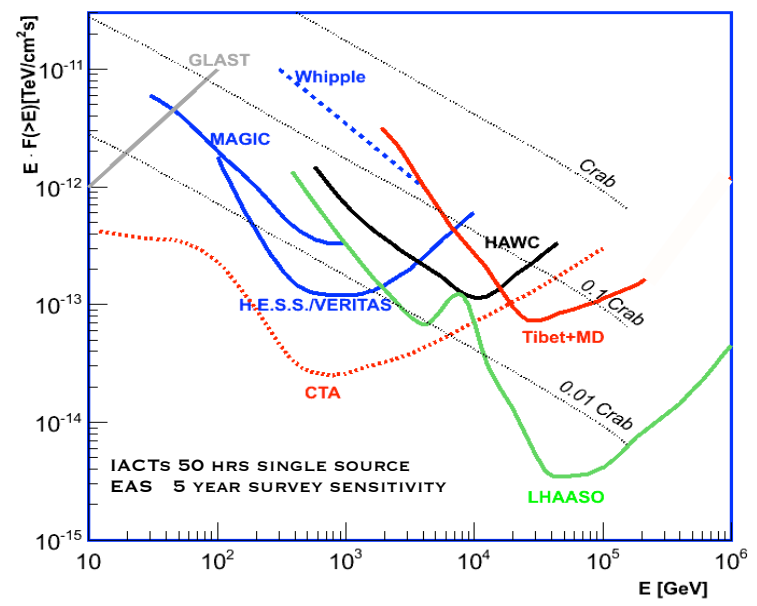

Fig. 1 Energy-flux sensitivities of current and future groundbased detectors - the IACT and EAS arrays in the energy range $10^{10}$ to $10^{15} \mathrm{eV}$ (courtesy of G. Sinnis).

\subsection{Future IACT Arrays}

The future of observational gamma-ray astronomy, at least for the next 10-15 years, is connected with the nextgeneration IACT arrays, first of all with the observatory CTA (Cherenkov Telescope Array) [4], cf. also Fig. 2.

The next generation of IACT arrays are aiming at (i) a significant (by an order of magnitude) improvement of the flux sensitivities in the standard 0.1-10 TeV energy interval ( $\mathrm{TeV}$ regime), and (ii) an expansion of the energy domain of IACT arrays in both directions - down to $10 \mathrm{GeV}$ (multi-GeV regime) and well beyond $10 \mathrm{TeV}$ (sub-PeV regime):

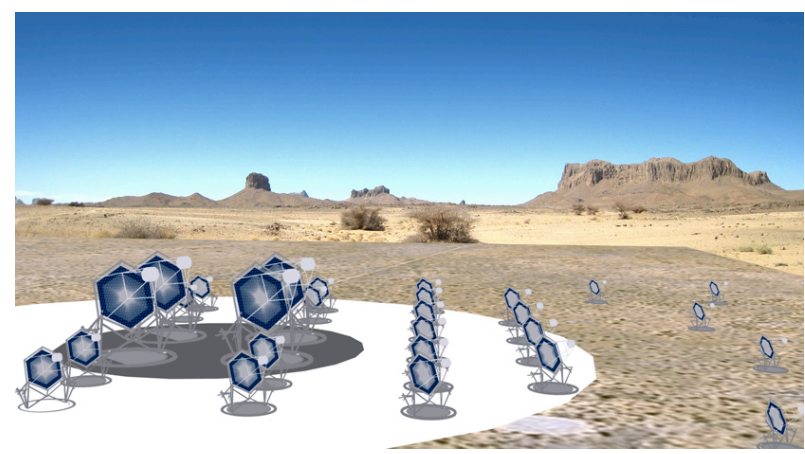

Fig. 2 Possible layout of the next-generation CTA instrument. From Ref. [4].

\section{- TeV regime:}

This is the "nominal" energy region where the IACT technique has achieved its best performance. The potential in this energy regime is still not saturated. With a stereoscopic array consisting of tens of 10 m-diameter (medium-size) class telescopes the minimum detectable energy flux could be reduced to the level of $10^{-14}$ $\mathrm{erg} / \mathrm{cm}^{2} \mathrm{~s}$, and the angular resolution be improved to $\delta \theta \leqslant 3$ arc minutes. Generally, the optimum distance between the telescopes is considered to be around $100 \mathrm{~m}$, the radius within which the Cherenkov light is distributed more or less homogeneously. However, if highest priority is given to the performance at energies around $1 \mathrm{TeV}$ and beyond, an increase of the distance between telescopes up to $300 \mathrm{~m}$ could be an attractive option. For a fixed number of telescopes this would increase the detection area by an order of magnitude, and, at the same time, improve the angular resolution to 1-2 arc minutes, although at the expense of a somewhat higher (by a factor of two or three) energy threshold. In any case, a reduction of the minimum detectable energy flux around $1 \mathrm{TeV}$ down to $10^{-14} \mathrm{erg} / \mathrm{cm}^{2} \mathrm{~s}$ seems to be a challenging but feasible "target". It will be a great achievement even by the standards of the most advanced branches of observational astronomy, allowing us to probe, in particular, potential $\mathrm{TeV}$ gamma-ray sources at luminosity levels of $10^{32}(d / 10 \mathrm{kpc})^{2} \mathrm{erg} / \mathrm{s}$ for galactic and $10^{40}(d / 100 \mathrm{Mpc})^{2} \mathrm{erg} / \mathrm{s}$ for extragalactic objects. Although for moderately extended sources, e.g. of angular size $\Psi \sim 1^{\circ}$, the minimum detectable energy flux will be by a factor of $\Psi / \delta \theta \sim 10-30$ higher, it would compete or be better than the energy flux sensitivities of the best current X-ray satellites, Chandra, $X M M$-Newton, INTEGRAL and Suzaku, and thus allow the deepest probes of non-thermal high energy phenomena in extended sources, in particular in shell-type SNRs, Giant Molecular Clouds, Pulsar-driven Nebulae (Plerions), Clusters of Galaxies, hypothetical Giant Pair Halos around AGN, etc. Such a system of 10-12 m diameter class IACTs with a field of view (FoV) of 6-8 degrees, 
will most likely constitute the core of the Cherenkov Telescope Array (CTA) - an initiative towards a major ground-based gamma-ray detector (see Fig. 2).

\section{- Sub-PeV regime:}

External and intergalactic absorption of gamma-rays, the limited efficiency of particle acceleration, the escape of highest energy particles from the source etc., result in a suppression of fluxes at the highest energies. The general tendency of decreasing gamma-ray fluxes with energy becomes especially dramatic above $10 \mathrm{TeV}$. Therefore, any meaningful study of cosmic gamma-rays beyond $10 \mathrm{TeV}$ typically requires detection areas as large as $1 \mathrm{~km}^{2}$. An effective and straightforward approach would be the use of IACT arrays optimised for detection of gamma-rays in the region up to $100 \mathrm{TeV}$ and beyond. This can be realised by modest, approximately $10-30 \mathrm{~m}^{2}-$ area reflectors separated from each other, depending on the scientific objectives and the configuration of the imagers, by 300 to $500 \mathrm{~m}$. The requirement on the pixel size of imagers is also rather modest, $0.25^{\circ}$ or so, however they should have large, up to 10 degree FoV for simultaneous detection of showers from distances more than $300 \mathrm{~m}$ [5]. A sub-array consisting of several tens of such small-size telescopes is included in the concept of CTA with a primary goal to study the energy spectra of gamma-ray sources well beyond $10 \mathrm{TeV}$. It will serve as a powerful tool for searches of galactic cosmic ray "PeVatrons", as well as nearby $(R \ll 10 \mathrm{Mpc})$ radio and starburst galaxies.

\section{- Sub-100 GeV regime:}

The energy threshold of detectors, $E_{\mathrm{th}}$, is generally defined as a characteristic energy at which the gamma-ray detection rate for a primary power-law spectrum with a photon index 2-3 achieves its maximum. It is known from Monte Carlo simulations as well as from the experience of operation of previous generation of IACTs, that in practice the best sensitivity is achieved at energies exceeding several times $E_{\mathrm{th}}$. Thus, for optimisation of gamma-ray detection around $100 \mathrm{GeV}$, one should reduce the energy threshold of telescopes to $E_{\mathrm{th}} \leqslant 30 \mathrm{GeV}$. This can be done by using very large, $20 \mathrm{~m}$-diameter (large-size) class reflectors. On the other hand, the reduction of the threshold to $30 \mathrm{GeV}$ is an important scientific issue in its own right; the intermediate interval between 30 and $300 \mathrm{GeV}$ could be crucial for certain classes of galactic and extragalactic gamma-ray sources. A sub-array consisting of several large-size telescopes as foreseen in CTA (see Fig. 2) will indeed significantly broaden the topics and scientific objectives of CTA.

Each of the IACT arrays discussed above covers at least two decades in energy with significant overlaps of the energy domains. Since these arrays contain the same basic elements, and generally have also common scientific motivations, an ideal arrangement would be if these sub-arrays are combined in a single facility which would have a sensitive and homogeneous coverage throughout the energy region from approximately $30 \mathrm{GeV}$ to 300 $\mathrm{TeV}$. The concept of CTA is based, to a large extent, on this argument [4]. The high detection rates, coupled with good angular and energy resolutions over four energy decades will make CTA a powerful multi-purpose gamma-ray observatory with a great capability for spectrometric, morphological and temporal studies of a diverse range of persistent and transient high-energy phenomena in the Universe.

\section{- Multi-GeV regime: Gamma-Ray Timing Explorers}

Despite the recent great achievements of high energy (HE) gamma-ray astronomy, there are obvious shortcomings in the performance of the current so-called "pairconversion" tracking detection technique - the most effective approach used in satellite-borne instruments for detection of gamma-rays at energies above $100 \mathrm{MeV}$. One should note that the flux sensitivity of Fermi-LAT at 1 $\mathrm{GeV}$ of about $10^{-12} \mathrm{erg} / \mathrm{cm}^{2} \mathrm{~s}$ can be achieved only after one year all-sky survey. While for persistent gamma-ray sources this seems to be an adequate sensitivity (given that a huge number of sources are simultaneously monitored within the large and homogeneous FoV), the small detection area significantly limits its potential, in particular for detailed studies of the temporal and spectral characteristics of highly variable sources like blazars or solitary events like gamma-ray bursts (GRBs). It will not be easy to improve the sensitivity achieved by FermiLAT at high energies by any future space-based mission, unless the Moon would be used in the (far) future as a possible platform for an installation of very large $\left(\gg 10 \mathrm{~m}^{2}\right)$ area pair-conversion tracking detectors. Apparently, the space-based resources of $\mathrm{GeV}$ gammaray astronomy have achieved a point where any further progress would appear extremely difficult and very expensive. In any case, for the next decades to come there is no space-based mission planned for the exploration of the high-energy gamma-ray sky. On the other hand, the principal possibility of an extension of the IACT technique towards $10 \mathrm{GeV}$ promises a new breakthrough in gamma-ray astronomy [1]. The (relatively) large gammaray fluxes in this energy interval, together with the huge detection areas offered by the IACT technique, can provide the highest gamma-ray photon statistics compared to any other energy band of cosmic gamma-radiation. Thus, in the case of a realization of $10 \mathrm{GeV}$-threshold IACT arrays, the presently poorly explored interval between 10 and $100 \mathrm{GeV}$ could become one of the most advanced domains of gamma-ray astronomy with a great potential for the studies of highly variable phenomena. 
The reduction of the energy threshold down to $10 \mathrm{GeV}$ or even less is principally possible within the basic concept of the IACT technique, but it requires an extreme approach of using $25 \mathrm{~m}$ diameter class telescopes with very high $(\geqslant 40 \%)$ quantum efficiency focal plane imagers to operate in a robotic regime at very high $(5 \mathrm{~km}$ or) mountain altitudes [6].

The energy range from several $\mathrm{GeV}$ to $30 \mathrm{GeV}$ has very specific astrophysical and cosmological objective: exploration of the highly variable non-thermal phenomena in the remote universe at redshifts of $\mathrm{z}=5$ (like large redshift quasars and GRBs), as well as the study of compact galactic sources such as pulsars and microquasars. A realization of such a gamma-ray timing explorer, hopefully during the lifetime of the Fermi observatory would be a great achievement for gamma-ray astronomy.

\section{TeV Sources}

\subsection{Supernova Remnants}

Massive stars are believed to end their life undergoing a supernova explosion. This explosion blows off their other layers into a supernova remnant (SNR), which heats the surrounding medium and accelerates cosmic-rays (electrons and protons) to extremely high energies. The radiation from shell-like SNRs consists of thermal emission from shock-heated gas and non-thermal emission from shock-accelerated particles. The theory of diffusive shock acceleration (DSA) at shock fronts $[7,8]$ predicts the production of a population of accelerated particles in SNRs that can interact with ambient magnetic fields, with ambient photon fields, or with matter. The amount of relativistic particles increases with time as the SNR passes through its free expansion phase, and reaches a maximum in the early stages of the Sedov phase. Correspondingly, the peak in gamma-ray luminosity typically appears some $10^{3}-10^{4}$ years after the supernova explosion.

In the TeV domain, presently seven shell-type SNRs Cas A [9-11], Tycho [12], SN 1006 [13], RX J1713.7-3946 [14,15], RX J0852-4622 (Vela Junior) [16], RCW 86 [17], and G353.6-0.7 (HESS J1731-347)[18] have been firmly identified as VHE gamma-ray emitters (see Table 1).

Remarkably, while the first six sources are well established young SNRs, the object G353.6-0.7 is the first SNR discovered serendipitously in $\mathrm{TeV}$ gamma-rays, and only later confirmed by radio and X-ray observations $[19,20]$. Moreover, a possible new SNR candidate, HESS J1912+101, has been postulated recently [21] based solely on its shell-type morphology at TeV energies, although no counterpart at lower energies has been detected so far. The two latest examples demonstrate the potential of large field-of-view Cherenkov telescopes for serendipitously discovering extended SNRs (of typical size $0.2-1^{\circ}$ at a distance up to $\sim 3.5 \mathrm{kpc}$ ). Their relatively large sizes and $\gamma$-ray luminosities of about $(0.1-1) \times 10^{33}$ $\mathrm{erg} / \mathrm{s}$ have enabled the detection of these objects up to distances of $\sim 3.5 \mathrm{kpc}$ (e.g., Tycho) with current instrument sensitivities (cf. Fig. 1). If the VHE gamma-ray luminosities detected from these objects reflect the typical luminosity of the SNR population in the Galaxy, future instrument like CTA should be able to detect SNRs up to $15 \mathrm{kpc}$, thus sampling the whole Galaxy. Taking the spatial distribution of SNRs in the Galaxy, their explosion rate, and the duration of the $\mathrm{TeV}$ emission (believed to last a few thousand years) into account, roughly $\sim 100$ new SNRs could be discovered at TeV energies [22] (in a naive approximation, without considering energy cut-offs, hard/soft spectral indices, etc.). Such an enlarged population would allow the study of these objects at different evolutionary stages, sampling their spectral energy distribution from a few hundred of $\mathrm{MeV}$ (with Fermi-LAT and AGILE) up to $100 \mathrm{TeV}$, in the cut-off regime.

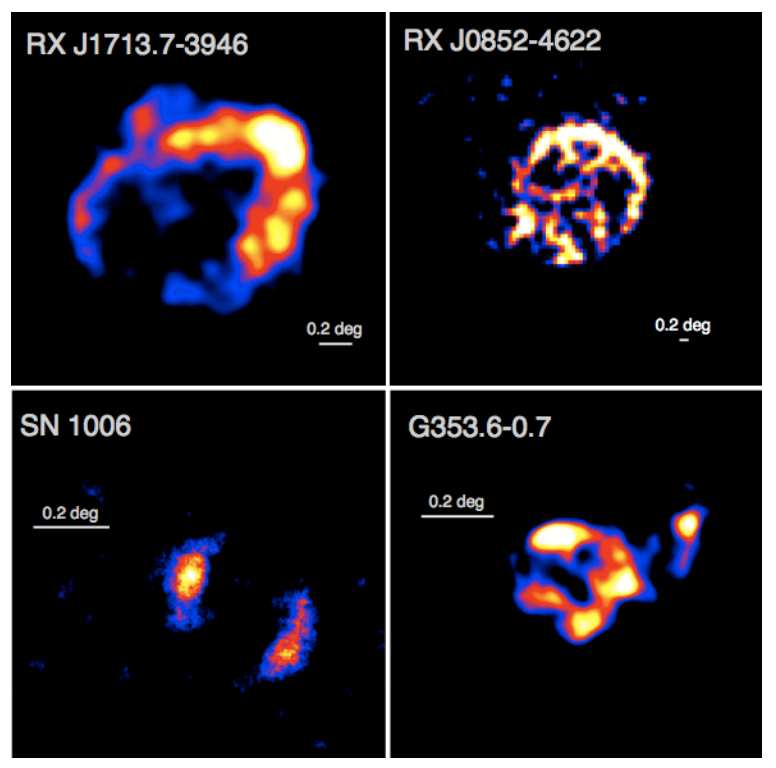

Fig. 3 Example of four shell-type SNRs detected at $\mathrm{TeV}$ energies with the H.E.S.S. instrument.

The sizes of several of these shell-like SNRs $\left(>0.1^{\circ}\right)$ has allowed to resolve them in VHE (see Fig. 3). The images of SNRs such as SN 1006, RX J1713.7-3946 or RX J0852-4622 have revealed a good correlation of the $\mathrm{TeV}$ emission sites with the non-thermal emission detected in X-rays, probing acceleration of relativistic particles up to multi-TeV energies. However, the relative contributions of accelerated protons and electrons to the gamma-ray production still remain unknown. The problem is that the ratio of gamma-rays produced by accelerated protons interacting with the surrounding gas, and by ultra-relativistic electrons up-scattering the $2.7 \mathrm{~K}$ 
Table 1: Shell-like SNRs firmly detected at TeV energies

\begin{tabular}{cccccc}
\hline \hline Name & Dist $(\mathrm{kpc})$ & Size $(\mathrm{pc})$ & Age $(\mathrm{yrs})$ & $\mathrm{L}_{\gamma}\left(10^{33} \mathrm{erg} / \mathrm{s}\right)$ & $\Gamma$ \\
\hline RX J1713.7-3946 & 1 & 17.4 & 1.6 & 8 & 2.0 \\
RX J0852-4622 & $0.2(1)$ & $6.8(34)$ & $0.4(5)$ & $0.26(6.4)$ & 2.2 \\
RCW 86 & $1(2.5)$ & $11(28)$ & $1.6(10)$ & $1(6)$ & 2.5 \\
SN 1006 & 2.2 & 18.3 & 1 & 1.24 & 2.3 \\
Cas A & 3.4 & 2.5 & 350 & 7 & 2.4 \\
Tycho & 3.5 & 6 & 438 & 0.1 & 1.95 \\
SNR G353.6-0.7 & 3.2 & 27 & $2.5(14)$ & 10 & 2.3 \\
\hline
\end{tabular}

CMB radiation, is very sensitive to generally unknown parameters, in particular to the gas density and the magnetic field of the ambient medium (cf., e.g. [302]). The efficiency of inverse Compton (IC) scattering is especially high at $\mathrm{TeV}$ energies (up to $\mathrm{E}_{\mathrm{e}} \approx 100 \mathrm{TeV}$, it proceeds in the Thomson regime, with a corresponding cooling time $\left.t_{\text {cool }}^{\mathrm{IC}} \propto 1 / \mathrm{E}_{\mathrm{e}} \propto 1 / E_{\gamma}^{1 / 2}\right)$. For example, the typical production time of a $1 \mathrm{TeV}$-photon by an electron and a proton of the same characteristic energy of about $20 \mathrm{TeV}$, are $\approx 5 \times 10^{4} \mathrm{yr}$ and $5 \times 10^{7}\left(\mathrm{n} / 1 \mathrm{~cm}^{3}\right)^{-1}$ yr, respectively (see, e.g. [23]). Correspondingly, at $1 \mathrm{TeV}$ the ratio of the production rates of IC to $\pi^{\circ}$ decay gamma-rays, is approximately $10^{3}\left(\mathrm{~W}_{\mathrm{e}} / \mathrm{W}_{\mathrm{p}}\right)(\mathrm{n} / 1$ $\left.\mathrm{cm}^{-3}\right)^{-1}$, where $W_{e}$ and $W_{p}$ are the total energies in $20 \mathrm{TeV}$ electrons and protons, respectively. Thus, even for a very small electron-to-proton ratio (at the stage of acceleration), $\mathrm{e} / \mathrm{p}=10^{-3}$, the contribution of the IC component will dominate over the $\pi^{\circ}$-decay gamma-rays (in the shell with a typical gas density $\mathrm{n} \leqslant 1 \mathrm{~cm}^{-3}$ ), unless the magnetic field in the shell significantly exceeds $10 \mu \mathrm{G}$. In this case, the accelerated electrons are cooled predominantly via synchrotron radiation, thus only a small fraction, $w_{\mathrm{CMB}} / w_{\mathrm{B}} \approx 0.1(\mathrm{~B} / 10 \mu \mathrm{G})^{-2}$, will be released in IC gamma-rays. Alternatively, the proton-to-electron acceleration ratio should exceed $e / p \sim 10^{3}$ which, in principle, cannot be excluded given the uncertainty associated with one of the key aspects of DSA related to the so-called injection problem (see [24]).

In cases like RX J1713.7-3946, Tycho or Cas A, the magnetic field has been estimated from multi-wavelength observations to be $>0.1 \mathrm{mG}[25,26]$, restricting the contribution of the IC emission and in principle favouring an hadronic origin of the $\mathrm{TeV}$ emission. Nevertheless, if the IC and synchrotron components of the radiation are formed in different zones, these constraints are less robust. For instance, a difference of the magnetic field in the upstream and the downstream region could result in a positional shift of the production regions of synchrotron $\mathrm{X}$-rays and IC gamma-rays, and more complex models implying multi-zone emission would need to be invoked $[27,28]$. In general, while the distribution of the X-ray radiation is dominated by the strength of the magnetic field, the $\mathrm{TeV}$ emission traces the particle distribution and does not depend on the magnetic field, allowing a more unbiased study of the particle acceleration in the shell. With the angular resolution of current instruments (of the order of $\approx 0.1^{\circ}$ ) those different sites are still indistinct, but the future improvement of the angular resolution to a few arcmin should permit a detailed study of the $\mathrm{TeV}$ radial profile in sources like RX J1713.7-3946 or SN 1006 in comparison with the X-ray radiation profile. The spectral energy distribution (SED) of these young SNRs extends over almost five decades, from a few hundred $\mathrm{MeV}$ to a few tens of TeV. At low energies the SED part for some of these TeV shell-like SNRs has been detected with the Fermi-LAT telescope [29-32]. The coverage of the spectrum at low energies has improved our understanding of the origin of the gamma-ray emission, but also evidenced a more complicated scenario in which different regions can contribute to the total emission, such as the reverse shock [28] or dense clouds embedded in the shock [33,34]. The photon spectra of Tycho, RX J0852-4622 and Cas A continues to the MeV-GeV range with a rather hard spectral index of $\simeq 2.0$ as predicted by the DSA theory [35-37]. This fact, together with the high magnetic field amplification derived from synchrotron X-ray filaments, preventing in principle a large IC contribution from leptons, favour an hadronic scenario in these SNRs. Moreover, high-energy radiation up to at least a few $\mathrm{TeV}$ has also been detected from these SNRs without an indication for a turnover in the spectrum. An extension of the high-energy emission by a factor two or three beyond $10 \mathrm{TeV}$ could only be explained through hadronic interactions, given the fast Klein-Nishina-cooling suffered by $100 \mathrm{TeV}$-electrons emitting in this energy regime, and would robustly exclude an IC origin of the radiation. It would also provide a definitive probe of SNRs as origin of the cosmic-ray sea (see Section 3.1.).

RX J0852-4622 and RXJ1713.7-3946, for which large magnetic fields have been estimated, face some difficulties when modeling their gamma-ray emission. These 
two SNRs have similar ages, sizes, and radio, X-ray and TeV gamma-ray spectra, although RX J1713.7-3946 shows a softer spectral index $(\simeq 1.5)$ in the $100 \mathrm{MeV}$ to $1 \mathrm{GeV}$-energy range, similar to the predicted indices in a leptonic scenario. In both cases, the apparent low gas density $\left(\mathrm{n} \simeq 0.1 \mathrm{~cm}^{-3}\right)[38]$ poses troubles to standard hadronic scenarios $[28,39,40]$. Still, even in the case of a very low gas density of the shell, the contribution of hadronic gamma-rays could be significant, if accelerated protons interact with the dense cores of molecular clouds embedded in the shell [82]. In such a case, slow diffusion could prevent low-energy particles to penetrate into these dense cores, suppressing the low-energy gamma-ray emission and naturally explaining the hard gamma-ray spectrum measured in RX J1713.7-3946. At the highest energies, RX J1713.7-3946 shows a energy cut-off above few $\mathrm{TeV}$, excluding $\mathrm{PeV}$ protons from this remnant. However, an escape of high-energy protons that cannot be confined in the shell, can not be excluded and might be a plausible explanation. In fact, at $\mathrm{GeV}$ energies, a large number of mid-age SNRs has been discovered, while only a small fraction of them shines at $\mathrm{TeV}$ energies. The gamma-ray emission in these cases is likely related to interactions of cosmic-rays with dense gaseous complexes [34]. In cases like W51C, detected up to $\sim 5 \mathrm{TeV}$ [41,42], an enhancement of the hadronic origin due to the large gas density in the region seems clearly favoured. On the other hand, the best example illustrating the escape of high-energy particles is the $10^{4}$ yr-old SNR W28 [43], where a clear correlation between the $\mathrm{TeV}$ emission and massive molecular clouds emitting in $\mathrm{CO}$ has been observed. Some of these clouds are also bright at $\mathrm{GeV}$ energies. Another example of this type of scenario is IC 443 [44-47], where the $\mathrm{GeV}$ and $\mathrm{TeV}$ emission appear shifted from each other. These images seem to support an escape scenario where, depending on the location of the massive clouds, the time of particle injection into the interstellar medium and the diffusion coefficient, a broad variety of energy distributions may be expected.

\subsection{Pulsars}

Pulsars - rapidly rotating and highly magnetised neutron stars surrounded by a rotating magnetosphere and accompanied by relativistic outflows - emit radiation at all wavelengths. Charged particles (electrons and positrons) are thought to be efficiently accelerated in the electromagnetic fields of the pulsar, producing $\gamma$ radiation via e.g. curvature processes and supporting the formation of a cold relativistic outflow beyond the light cylinder. This pulsar wind carries almost the entire rotational energy of the pulsar in the form of Poynting flux and/or kinetic energy of the bulk motion, and creates a standing shock wave (the termination shock) when it in- teracts with the ambient medium. Particles accelerated at this shock are responsible for the steady and usually very extended non-thermal radiation observed (see Sec. 2.3).

Although pulsars have been traditionally a subject of radio astronomy, with $\approx 1800$ pulsars found beaming radio waves, most of their radiation is emitted at highenergies (a few percent of their spin-down power). Indeed, in the last three years, the number of gamma-ray pulsars has increased exponentially from half a dozen to more than 150 [48] thanks to the new sensitive instruments Fermi-LAT and AGILE. Despite the high Galactic background, the periodic gamma-ray emission stands out due to the high fluxes, hard spectral index and powerful timing analysis tools. The large statistics and good data quality has provided new insights into the physics of pulsars. In general, it is believed that the pulsed, periodic gamma-ray radiation originates in regions of the magnetosphere, called gaps, where the electric field has a parallel component along the magnetic field lines. This electric field efficiently accelerates electrons and positrons to relativistic energies causing them to emit synchro-curvature radiation in the form of gamma-rays. There are currently a few models that differ, primarily, on the location of these gaps [49-51], which are capable to explain the light-curves and spectral energy distributions. Other mechanisms have also been suggested such as a magnetosphere with a force-free structure [52] or a striped wind topology [53]. The Fermi-LAT-measured light curves and energy spectra indicate that gamma-ray emission from the brightest pulsars is produced in the outer magnetosphere with fan-like beams scanning over a large portion of the celestial sphere. The energy spectra for most of the gamma-ray pulsars are best described by a power-law function with an exponential cutoff of the form $\mathrm{E}^{-\Gamma} \exp \left[-\left(E / E_{0}\right)^{b}\right]$ with $b \leqslant 1$, and cut-off energy $E_{0}$ between 1 and $10 \mathrm{GeV}$ [48]. The detection of gamma-rays beyond a few $\mathrm{GeV}$ without indication for a super-exponential attenuation (i.e., $b>1$ ) effectively excludes the so-called polar cap model and gives a preference to models of gamma-ray production in the outer magnetosphere (in order to avoid severe pair-production in the strong magnetic field in low-altitude zones). Most of the measured spectra can be well-fitted with a simple exponential attenuation $(b=1)$ [48], which is in general well-explained by the mechanism of curvature radiation. However, an extension of the spectral measurements for the brightest gamma-ray pulsars towards both, higher and lower energies, has revealed that the spectra beyond the cut-off could be smoother $(b \simeq 0.5)$. For example, the phase-averaged spectrum of the Crab pulsar is better fitted with the combination of parameters $b=0.43, \Gamma=1.59$ and $E_{0}=0.50 \mathrm{GeV}$ [54], rather than $b=1, \Gamma=1.97$ and $E_{0}=5.8 \mathrm{GeV}$ as reported earlier by the Fermi-LAT collaboration based 
on smaller gamma-ray statistics [55]. In any case, if the above noted fit of the energy spectra is extrapolated to higher energies, a dramatic decrease of gamma-ray fluxes well beyond $10 \mathrm{GeV}$ is expected, preventing the detection of pulsed emission with the current instrument at $\sim 100 \mathrm{GeV}$. The MAGIC telescope, using a novel trigger system detected sub- $100 \mathrm{GeV}$ pulsed emission from the Crab pulsar [56], favouring models with exponential or sub-exponential cut-offs (slot gap and outer gap models).

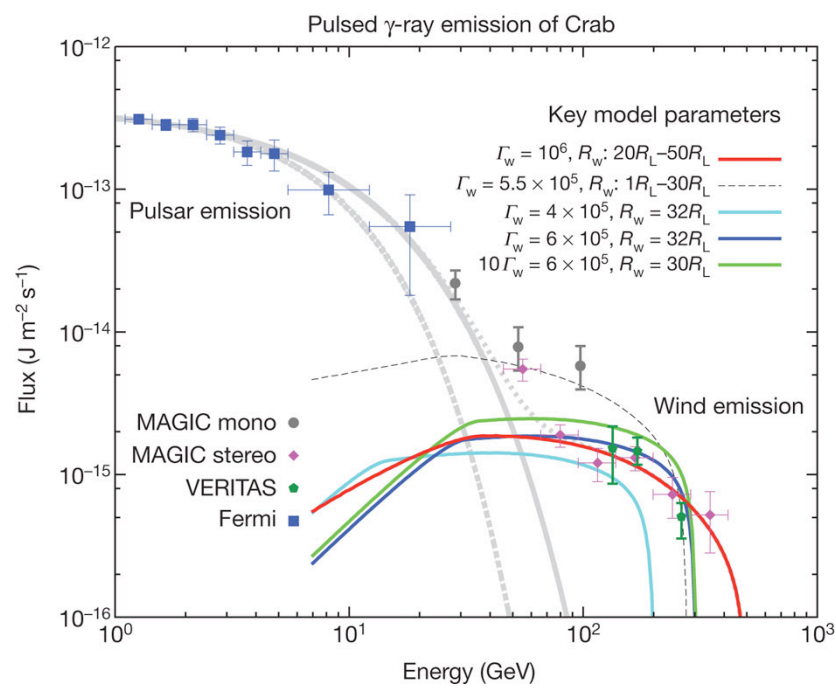

Fig. 4 Spectral energy distribution (SED) of the pulsed gammaray emission from the direction of the Crab pulsar and nebula. Fermi-LAT points are shown (blue squares) together with MAGIC (grey and pink) and VERITAS (green) points. A Fermi-LATpoints best-fit, using two different hypotheses $\left(b=1, E_{0}=5.8\right.$ $\mathrm{GeV}$ and $\Gamma=1.97$ and $b=0.85, E_{0}=7 \mathrm{GeV}$ and $\left.\Gamma=1.97\right)$, is displayed in grey. The pulsed VHE radiation can be successfully accounted for (light blue, blue, green and red curves) by inverse Compton up-scattering of the pulsed magnetospheric X-ray emission by a cold ultra-relativistic pulsar wind (see Sec. 3.2). From Ref. [60].

Yet unexpectedly, pulsed $\gamma$-ray emission above $100 \mathrm{GeV}$ and up to $400 \mathrm{GeV}$ of unknown origin was recently detected from the Crab with the VERITAS and MAGIC telescopes [57,58], cf. Fig. 4, challenging models for the origin of the periodic emission in neutron stars. Different explanations could be pursued to accommodate these new experimental findings within current models, such as secondary emission of electrons in the outer magnetosphere [59] or IC emission from energetic electrons in the ultra-relativistic pulsar wind [60] (cf. also Sec. 3.2). Approaches like these predict different spectral shapes and light-curve behaviour at $\mathrm{GeV}$ and $\mathrm{TeV}$ energies. The detected phase-averaged, pulsed emission (Fig. 4) could in principle be fitted by extrapolating the reported Fermi fluxes to the VHE domain as a power law with photon index of $3.8 \pm 0.5$ and a flux of $1 \%$ of the flux of the Nebula at $150 \mathrm{GeV}$, but the nature of such an extrapo- lation seems rather difficult to justify on physical (magnetospheric) grounds [60]. The VHE light curve shows a double peak structure well-aligned with the light curve at lower energies, although narrower by a factor of two or three than those measured by Fermi-LAT. The spectrum of the narrow peaks, extending no more that $10 \%$ of the rotational period, does not show a significant deviation in its shape from the global spectral fit. Assuming a common (magnetospheric?) origin, a smooth connection of the VHE points with the HE points can be achieved by fitting the data with a broken power-law function, but to the exclusion of an exponential cut-off. An alternative explanation consists in considering the entire gamma-ray region as a superposition of two separate components, a nominal (magnetospheric) $\mathrm{GeV}$ one and an additional VHE component produced by IC up-scattering of the magnetospheric emission by the fast pulsar wind [60]. Measuring the spectral shape with high precision in the near future will provide constrains on these models and allow to investigate the connection with the low-energy points around $50 \mathrm{GeV}$ and the spectral extension above $400 \mathrm{GeV}$. Up to now, the observed $\gamma$-ray features make the Crab a unique source of this kind at VHE. An increase of the sample by observing the brightest FermiLAT pulsars, such Vela or Geminga will be pursued by H.E.S.S. II, MAGIC II and VERITAS (and CTA in the future), providing more input to understand the origin of this pulsed VHE radiation [61].

\subsection{Pulsar Wind Nebulae}

Relativistic winds from energetic pulsars carry most of the rotational power into the surrounding medium, accelerating particles to high energies, either during their expansion or at the shocks produced in collisions of the winds with the sub-sonic environment. Accelerated leptons can interact with magnetic fields and low-energy radiations fields of synchrotron, thermal or microwavebackground origins. As a result, non-thermal radiation is produced from the lowest possible energies up to $\simeq 100$ $\mathrm{TeV}$. For magnetic fields of few $\mu \mathrm{G}$, freshly injected electrons (and positrons) create a synchrotron nebula around the pulsar, ranging from the radio to the X-ray and, in some cases, to the $\mathrm{MeV}$ band. At high energies a second component appears as a result of Comptonization of these soft photon fields by the relativistic leptons, creating an extended IC-nebula around the pulsar [56]. 


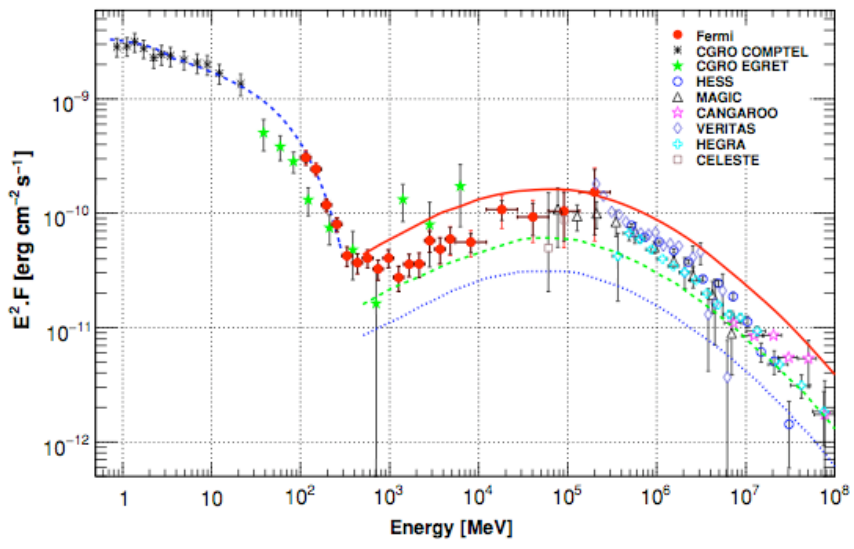

Fig. 5 Spectral energy distribution (SED) of the Crab Nebula in the high- and very high energy gamma-ray domain. The spectral points from low to VHE gamma-rays are shown together with a fit of the synchrotron component (blue dashed line) and predictions for IC gamma-rays calculated for three different values of the mean magnetic field: $B=100 \mu \mathrm{G}$ (solid red line), $B=200 \mu \mathrm{G}$, and the equipartition field of the nebula of $300 \mu \mathrm{G}$. From Ref. [55], reproduced by permission of the AAS.

VHE observations of these pulsar wind nebulae (PWNe) have revealed PWNe to be the most effective Galactic objects for the production of VHE gamma-rays, allowing the detection of such systems even outside our own Galaxy (in the LMC [63]). As recently as of 2004, only the Crab PWN was detected with a steady gamma-ray flux above $1 \mathrm{TeV}$ of $\left(2.1 \pm 0.1_{\text {stat }}\right) \times 10^{-11} \mathrm{~cm}^{-2} \mathrm{~s}^{-1}[64,65]$. The development of the new sensitive IACTs in the last years has raised the number of likely PWNe detected to at least 27 sources, whereas many of the unidentified gamma-ray sources are widely believed to be PWNe (or old relic $\mathrm{PWNe}$ ) [23].

For many years, the Crab nebula was considered as a standard candle for the cross-calibration of VHE detectors, as the brightest persistent point-like TeV gammaray source seen effectively from both hemispheres. The main features of its non-thermal emission, extending over 21 decades of frequencies, has been satisfactorily described by the formation of a PWNe based, to a large extent, on a simple MHD model for the interaction of a cold ultra-relativistic electron-positron wind with the interstellar medium [66]. Recent detailed two-dimensional MHD simulations $[67,68]$ have confirmed such a concept, at least for the Crab Nebula. The IC emission detected at $\mathrm{TeV}$ provides crucial information about the conditions in the nebula even when it only constitutes a small fraction of the synchrotron luminosity of the nebula. In particular, a comparison of the X-ray and $\mathrm{TeV}$ gamma-ray fluxes observed from the Crab Nebula has lead to a robust estimate of the average nebular magnetic field of less than $100 \mu \mathrm{G}$, in good agreement with predictions for the termination of the wind in MHD theory [66]. Figure 5 shows the high-energy coverage of the Crab Neb- ula spectrum. While the COMPTEL and EGRET data carry information about the synchrotron radiation in the cut-off region, the Fermi-LAT data reveal the sharp transition from the synchrotron to the IC component at around $1 \mathrm{GeV}$. At an energy $\mathrm{E} \simeq 100 \mathrm{GeV}$, a clear indication of the IC maximum is supported by both satellite (Fermi-LAT [55], and ground-based (MAGIC [69] and VERITAS [58]) measurements, which show remarkable agreement with each other. The measurements with ground-based IACTs have almost approached $100 \mathrm{TeV}$ $[64,70,71]$, where the IC component should still extend to the energy region set by the maximum energy of the accelerated electrons, i.e., $1 \mathrm{PeV}$. Although the production of gamma-rays at such energies takes place in the KleinNishina regime, and is therefore strongly suppressed, future instrument such CTA should be able to detect this emission.

Yet, despite the large coverage and deep observations, many aspects of this unique source are still unresolved. For instance, rapid high-energy flares with rise time as short as 6 hours from the Crab PWN have been reported by the Fermi-LAT and the AGILE collaboration [54,72]. This amazing discovery has opened new questions such as how these flares connect with the pulsar energy release or as to their origin (are they related to the inner pulsar wind or to the magnetosphere?, see e.g., [7377]). The exceptionally high fluxes during the active state in April 2011 allow detailed spectroscopy for different flux levels [54]. In order to study the spectral evolution of the flaring component, a steady-state (constant) background has been assumed with a steep powerlaw spectrum described by a photon index $\Gamma_{\mathrm{b}}=3.9$. The spectrum of the flaring component has been assumed in the form of power-law with exponential cutoff, $\nu F_{\nu}=f_{0} E^{2-\Gamma_{\mathrm{f}}} \exp \left[-\left(E / E_{0}\right)^{\kappa}\right]$. The results show that the spectra during all selected windows can be well described by the same photon index $\Gamma_{\mathrm{f}}=1.27 \pm 0.12$ and exponential cutoff index $\kappa=1$, but with variable total flux $f_{0}$ and the cut-off energy $E_{0}$. A variation by a factor of two allows a good fitting of the data, but the total flux has to be changed more than an order of magnitude in this approach. While different theories (including synchrotron radiation and reconnection) have been put forward to explain these flares, many key issues are still unresolved.

Even as one of the strongest sources in the TeV sky, the Crab nebula is very inefficient in producing gammarays through IC scattering, and only its extremely high spin-down power compensates for this. The energy density of the magnetic field (of the order of $\sim 100 \mu \mathrm{G}$ ) exceeds by more than two orders of magnitude the radiation energy density. Thus, less than one per cent of the energy of the accelerated electrons is released in IC gamma-rays, the rest being emitted through synchrotron radiation. In other systems, the pulsar wind is not as 
powerful as the one in Crab, resulting in weaker magnetic fields in the nebula of the order of a few $\mu \mathrm{G}$. This low magnetic field translates into a more efficient emission via IC at VHE due to the sharing of the electron energy losses between synchrotron and IC mechanism. For instance, in the case of the cosmic microwave radiation $(\mathrm{CMB})$, the two radiation components are related through $L_{\gamma} / L_{\mathrm{X}}=w_{\mathrm{CMB}} / w_{\mathrm{B}} \simeq 1(B / 3 \mu \mathrm{G})^{-2}$. This implies that in a PWN with a nebular magnetic field of about $10 \mu \mathrm{G}$ or less, the IC gamma-ray production efficiency could be as large as $10 \%$. Given that the rotational energy of pulsars is eventually released in relativistic electrons accelerated at the termination shock, PWNe associated with young pulsars with spin-down luminosities $L_{0} \geqslant 10^{34}(\mathrm{~d} / 1 \mathrm{kpc})^{2} \mathrm{erg} / \mathrm{s}$ were expected to be detected [78]. These expectations have been confirmed by the results obtained with MAGIC and VERITAS, but overall by the survey performed with H.E.S.S. The Galactic plane survey (GPS) as seen by H.E.S.S. in Fall 2012 is shown in Fig. 6. The survey, covering a range between $\left[-85^{\circ}, 60^{\circ}\right]$ in longitude and $\left[-2.5^{\circ}, 2.5^{\circ}\right]$ in latitude, has revealed more than fifty new VHE $\gamma$-ray sources, out of which more than half are believed to be gamma-ray PWNe, located in the close vicinity of young and energetic pulsars.
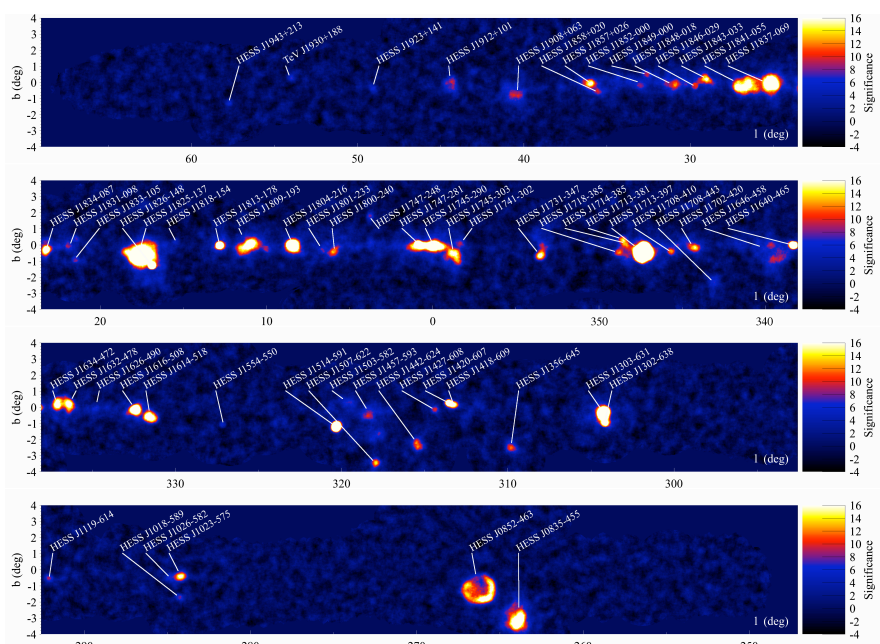

Fig. 6 Significance (pre-trial) map of the Galactic plane survey by H.E.S.S. From Ref. [296].

Presently PWNe constitute the largest galactic TeV source population. Many previously dubbed "dark" TeV gamma-ray sources, including the first unidentified $\mathrm{TeV}$ gamma-ray source discovered by the HEGRA collaboration, TeV J2032+4130 [79], have later been identified as PWNe. Most of these identifications with PWNe are quite convincing, yet still tentative, except for several ones which are firmly identified, either by excellent radio/X-ray morphological correlations, such as the Kookaburra complex, MSH 15-52 and VelaX [80,81], or by observations of an energy-dependent morphology, tracing the cooling mechanisms in the leptonic population injected by the pulsar (as observed in HESS J1825137 or HESS J1303-631 [82,83], cf. Fig. 7).

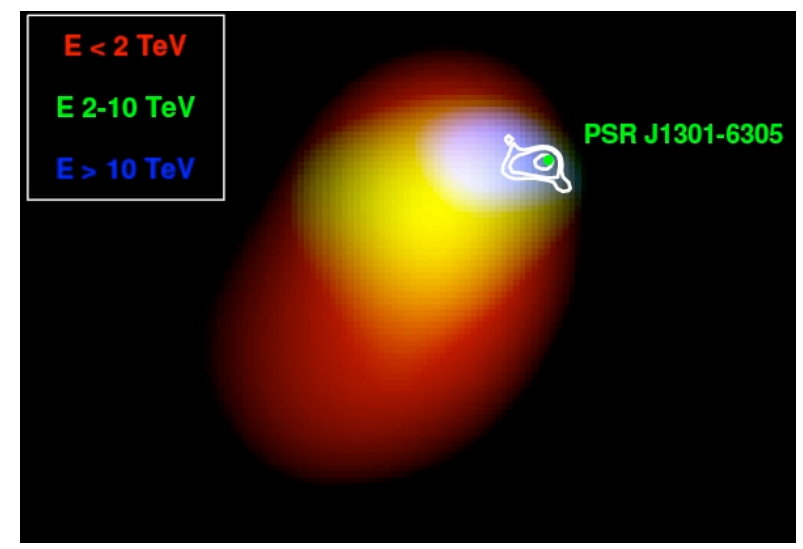

Fig. 7 VHE image of the $\mathrm{TeV}$ pulsar wind nebula candidate HESS 1303-631 at different energy ranges. The highest-energy photons originate near to the pulsar. X-ray (XMM) contours are shown in white. See Ref. [297]

Out of the PWNe detected at VHE two different populations of PWNe seem to be emerging: PWNe associated to young, compact X-ray PWNe, often still embedded in their associated supernova remnant; and evolved (extended and resolved) sources, in which the TeV emission seems to be due to a "relic" population of electrons, whereas the associated shell has already faded away. In the latter group, the centre of gravity of the extended $\mathrm{TeV}$ images is often offset with respect to the position of the powering pulsar. Asymmetric, one-sided images of these PWNe have also been found in X-rays, but on significantly smaller scales. Although the mechanism which causes PWN offsets from the pulsar positions is not yet firmly established, this effect could be linked to the propagation of a reverse shock created at the termination of the pulsar wind in a highly inhomogeneous medium [62]. The significantly larger extension of the $\mathrm{TeV}$ emission region can be understood as a result of several factors: (i) Generally, for PWNe with magnetic field of order of $10 \mu \mathrm{G}$ or less, as apparently the case for most TeV PWNe, the electrons responsible for the Xray emission are more energetic than the electrons emitting TeV gamma-rays. Therefore, synchrotron-burning of the highest-energy electrons results in a smaller size of the X-ray source. (ii) When electrons diffuse beyond the PWN boundary, they emit less synchrotron radiation (due to the reduced magnetic field), but they can still effectively radiate gamma-rays via inverse Compton scattering of the universal CMB. (iii) Finally, because of the high X-ray background, the sensitivities of X-ray detectors like Chandra and XMM-Newton are dramatically reduced beyond several angular minutes. This significantly limits the potential of these instruments for weak, 
extended X-ray sources. In contrast, the sensitivity of IACT arrays remains almost unchanged approximately within a $1^{\circ}$ radius of field-of-view. This flat response makes IACT technique the most powerful tool for studying the non-thermal population of electrons in PWNe.

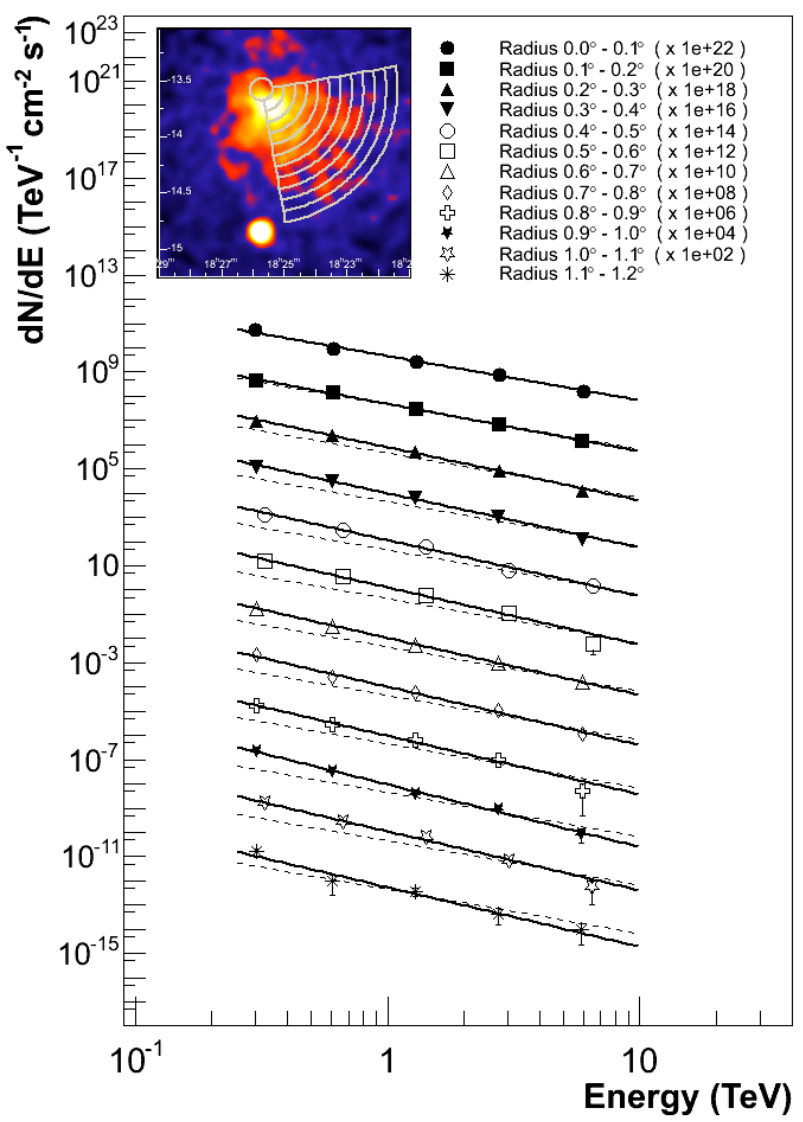

Fig. 8 Energy-dependent VHE morphology of pulsar wind nebula HESS J1825-137, showing a softening of the spectra with increasing distance from the pulsar. The plot shows the energy spectra in radial bins as indicated in the inset (with the dashed line from the innermost region for comparison). From Ref. [82]

The asymmetry observed in those PWNe has been explained as a consequence of the propagation of the precursor supernova explosion in the inhomogeneous interstellar medium [84], resulting in a faster evolution of the associated PWN in the opposite direction of the denser environment or/and a high kick-off velocity of the pulsar, displacing it from the centre of the supernova explosion. The accumulation of particles with time, the continuous injection and the ubiquitous presence of a soft photon target $(\mathrm{CMB})$ make these objects extremely efficient in the production of VHE emission. The high flux and extension of these TeV PWNe have permitted the investigation of the spectral behaviour with good statistics in different regions of the nebula, unveiling a softening of the gamma-ray spectral index as a function of the distance from the pulsar (see Fig. 8). This effect is due to the radiation of uncooled electrons which quickly leave the compact region near the pulsar, suffering significant radiative losses as they propagate away. This seems also to be the case for Vela X, a nearby PWN related to the powerful pulsar PSR J0835-4510 ( $\tau \approx 11,000 \mathrm{yr}$, $\left.L_{0}=7 \times 10^{36} \mathrm{erg} / \mathrm{s}\right)$. Vela $\mathrm{X}$ has been established [81] as one of the strongest $\mathrm{TeV}$ gamma-ray sources in the Galaxy. The energy spectrum of this source is quite different from other galactic sources; it is very hard at low energies, with photon index $\Gamma \approx 1.5$, and contains a high-energy exponential cut-off resulting in a distinct maximum in the SED at $10 \mathrm{TeV}$. Because of the nearby location of the source $(d \approx 300 \mathrm{pc})$ we see, despite the large angular size of the gamma-ray image of order of 1 degree, only the central region with a linear size less than several pc. In this regard, Vela $\mathrm{X}$ is a perfect object for the exploration of processes in the inner parts of the nebula close to the termination shock. The significantly improved sensitivity of the future CTA instrument and its superior angular resolution (one to two arc minutes at $10 \mathrm{TeV}$ ) should allow a unique probe of the relativistic electrons inside the region of the termination shock, i.e., at the very heart of the accelerator.

Along with these evolved nebula, a large number of compact objects have also been identified recently (see, e.g. $[85,86])$, in which the PWN is still expanding within the shell. A text-book example is the composite SNR G327.1-1.1 (HESS J1554-550) [87], in which the detected $\mathrm{TeV}$ emission is spatially coincident with the Xray and radio $\mathrm{PWN}$, well inside the remnant. A similar case is the newly detected source HESS J1818-154 [88], embedded in the SNR G15.4+0.1. The latter was discovered after a long exposure of $145 \mathrm{~h}$ with a flux of $1.5 \%$ of the Crab Nebula flux, and no X-ray or radio PWN has been detected yet, allowing SNR G15.4+0.1 to be identified as a composite SNR by means of VHE observations only. Those objects display a very low magnetic field in comparison to the Crab Nebula of the order of a few $\mu \mathrm{G}$, compensating so the lower spin-down power luminosity with a particle-dominated wind, which allows an enhancement of the inverse-Compton emission at very high energy.

\subsection{TeV Binary Systems}

The number of TeV binary systems - sources emitting variable, modulated VHE emission composed of a massive star and a compact object - has increased steadily in the last years, thanks to the large time coverage and the deep and uniform exposure of the Galactic plane by MAGIC, VERITAS and H.E.S.S. The TeV emission is believed to arise from the interactions between the two objects, either in an accretion-powered jet (microquasar scenario), or in the shock between a pulsar wind and a stellar wind (wind-wind scenario) (see e.g. [89-93], cf. also Sec. 3.2). In the microquasar scenario, particle 
acceleration takes place in a jet which originates from an accretion disk. This scaled-down version of an active galactic nucleus opens the possibility to obtain significant insights into the mechanism of jet production. In the wind-wind scenario, on the other hand, particle acceleration occurs in the interaction region between a ultra-relativistic pulsar wind and the dense radiation field provided by the companion star. Likewise, X-rays and high-energy components are expected due to radiative (synchrotron and inverse-Compton) cooling of relativistic electrons accelerated at the termination shock $[94,95]$.

Four periodic binary systems have been firmly identified at VHE (PSR B1259-63 [96], HESS J0632+057 [97,98], LS 5039 [99] and LSI +61 303 [100-102]), whereas two more sources (HESS J1018-589 [103] and Cyg X-1 [104]) are less certain and still pending confirmation. The observed variability implies a compact emission region which translates into a point-like source morphology at a distance of 1 to $5 \mathrm{kpc}$. Indeed, the majority of pointlike sources detected in the H.E.S.S. Galactic Survey have been identify as TeV binary systems. This univocal identification is based on the observed VHE variability/periodicity and correlations with flux variation at other wavelengths. They exhibit a maximum flux of $\sim 5-15 \%$ of the Crab Nebula flux and apparent similar spectral indices (2.0 to 2.7), but the enlargement of the $\mathrm{TeV}$ (and $\mathrm{GeV}$ ) binary sample has indicated a very diverse behaviour from one system to the other, demanding a detailed source-to-source investigation.

The first TeV binary established was the pulsar-B2Ve star system associated to PSR B1259-63 (or LS 2833) in 2004, which was anticipated before its detection [95]. In this system, a $48 \mathrm{~ms}$ pulsar is moving around a massive Be star, crossing its disk every 3.4 years, on a highly eccentric $(\mathrm{e}=0.87)$ orbit. The observations show a complex light curve, and the VHE emission can be satisfactorily explained in a pulsar-wind stellar-wind scenario, although the different year-to-year observations still challenge current models. Moreover, the source exhibited a large post-periastron orphan flare at $\mathrm{GeV}$ energy that was not observed in the $\mathrm{TeV}$ range $[105,106,290]$, which lasted approximately two weeks with an enhanced flux above $100 \mathrm{MeV}$ at the level of $3 \times 10^{-10} \mathrm{erg} \mathrm{cm}^{-2} \mathrm{~s}^{-1}$. Several scenarios have been proposed to account for this phenomenon, involving energy-dependent absorption processes and/or Comptonization of the photon field provided by the star by the cold ultra-relativistic pulsar wind [107].

The second, very-long-period ( $\sim 320$ days)-system was discovery serendipitously in the H.E.S.S. survey, being one of the very few point-like $\left(<2^{\prime}\right)$ unidentified sources. HESS J0632+057 was finally identified in a joint H.E.S.S. and VERITAS campaign, followed up by MAGIC observations, through long-term X-ray observations with
SWIFT, that succeeded in confirming its nature. Strong evidence for periodic X-ray variability with a very long period of $321 \pm 5$ days has been reported [108], implying the discovery of a binary for the first time on the basis of $\mathrm{TeV}$ observations (see also Fig. 9).

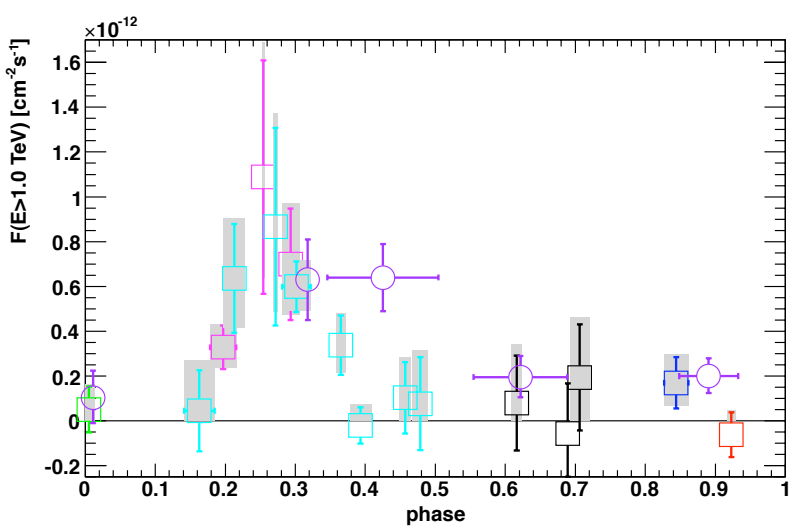

Fig. 9 VHE observations of the binary system HESS J0632+057 folded with a period of 321 days. The H.E.S.S. (circular markers) and VERITAS (open squares) measurements are shown in different colours for different observational periods. From Ref. [97].

The last two mentioned VHE binary systems, LSI +61303 and LS 5039, show short-periodic orbital variability, of the order of days, allowing a larger integration of VHE data and deeper investigation of their light curve. However, they behave quite differently from each other. While LS 5039 (P 3.9 days) exhibits are very regular light curve, LSI+61 303, with a period of $\sim 26.5$ days, shows a quite erratic behaviour, likely related with a 1667 super-orbital variability [109]. The nature of the compact object for both system is unknown: It could be anything from a $1.4 \mathrm{M}_{\circ}$ neutron star to a (3.7) $4 \mathrm{M}_{\circ}$ black hole. No pulsation has been found in radio or Xray searches. It seems likely, however, that any pulsed radiation would be absorbed in the optical-thick dense ambient due to Compton scattering [22].

These two binary systems have also been detected with the Fermi-LAT telescope above $100 \mathrm{MeV}$. The spectrum of LS 5039 shows a clear hardening in the 0.3 to $20 \mathrm{TeV}$ region (see Fig. 10), while the $\mathrm{GeV}$ component shows a softening in inferior conjunction. On the other hand, at superior conjunction an opposite behaviour is observed. LSI +61303 on the contrary, does not show variation of the spectral index, but its emission vanished after October 2008, reappearing again in 2010, accompanied by a change in the high-energy flux with decrease of the orbital modulation in 2009 [111-113]. From the multiwavelength data it is clear that more sophisticated scenarios are needed to understand the acceleration and emission processes involved in these two sources.

Finally, two more VHE regions have been associated with binary systems: MAGIC has reported a $4 \sigma$ evidence for VHE emission from the direction of the Microquasar 

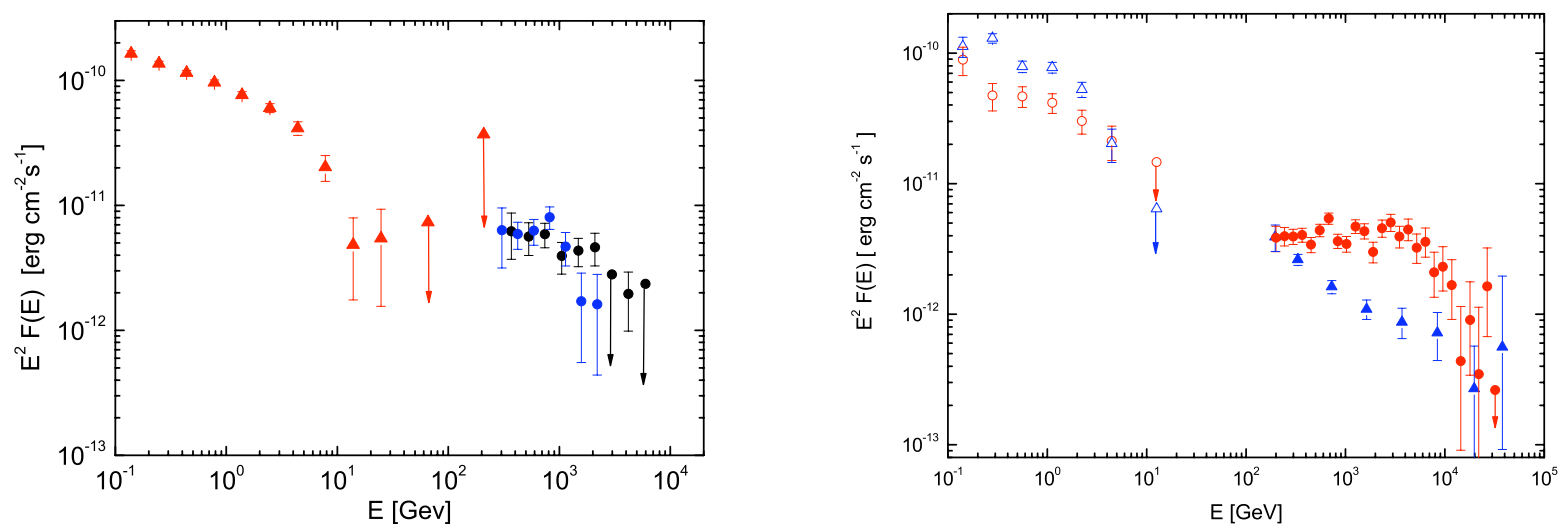

Fig. 10 High-energy (Fermi-LAT) and VHE (MAGIC, VERITAS and H.E.S.S.) observations of LSI +61 303 (left) and LS 5039 (right), cf. Refs. $[298,299]$. The right figure shows the spectral data at inferior conjunction in red circles whereas the observations in the superior conjunction are shown in blue triangles.

Cyg X-1 [104], correlated with an increase in soft and hard X-rays, but this was not confirmed during later, similarly high X-ray flux flaring events; and the GeV 16.5 days binary system 1FGL J1018.6-5856 [114], coincident with the H.E.S.S. source HESS J1018-589. For the latter, no VHE variability has been discovered yet, making the association somewhat unclear. Deep observations and uniform exposure in time with H.E.S.S. will help to clarify the origin of its VHE emission.

\subsection{Galactic Centre}

The Galactic Centre (GC) harbours many remarkable objects, including a few potential sites for particle acceleration and gamma-ray production, in particular the compact radio source Sgr A*, a suspected super-massive black hole located at the dynamical centre of the Galaxy. The GC contains a strong gamma-ray source (cf. Figs. 11 and 12) with a broad-band spectrum that spans from $100 \mathrm{MeV}[115]$ to $30 \mathrm{TeV}$ [116]. Assuming that gammarays from the entire interval are linked to the same source, the spectrum has an interesting form with several distinct features: Hard at low energies, with a photon in$\operatorname{dex} \Gamma \approx 2.2$, it becomes significantly steeper by $\Delta \Gamma \approx 0.5$ above $2 \mathrm{GeV}$ [115], then hardens again at $\mathrm{TeV}$ energies with a photon index $\Gamma \simeq 2.1$ and an apparent break or cut-off above $10 \mathrm{TeV}$ (see Fig. 12).
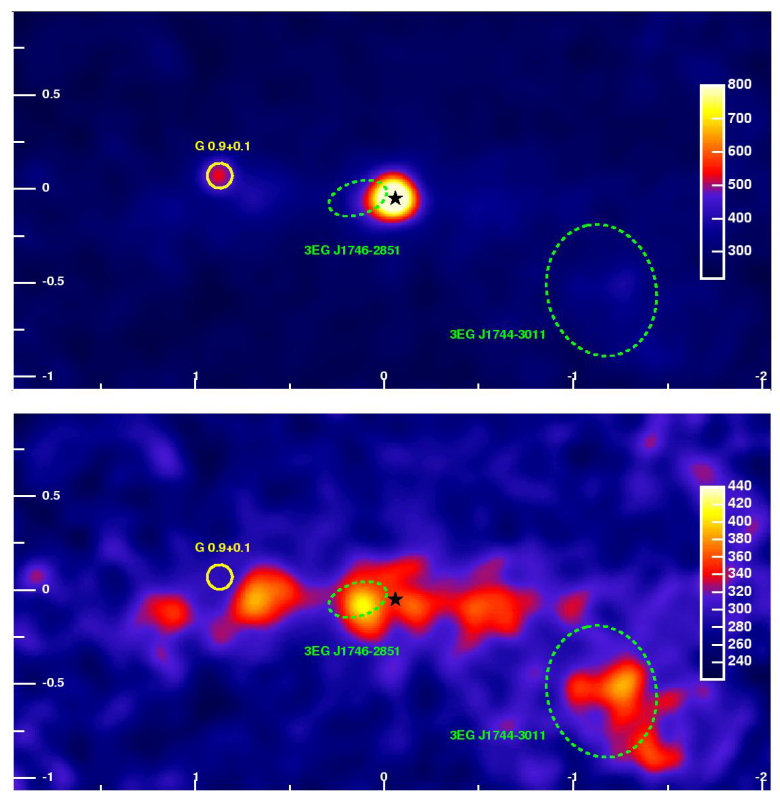

Fig. 11 The image of the several-hundred parsec region of the Galactic Centre in TeV gamma-rays (top: $\gamma$-ray count map; bottom: same map after subtraction of the two point sources). It contains a point like source (angular radius less than a few arcminutes), the gravity centre of which coincides with an accuracy of 13 arc-seconds with the compact radio source Sgr A* (marked with black star) - a supermassive black hole at the dynamical centre of the Milky Way [120,121]. The second point-like source located about one degree away positionally coincides with the composite supernova remnants G09+0.1 [85]. A prominent feature of this region is the ridge of diffuse emission tracing several well-identified giant molecular clouds (lower panel; cf. Ref. [122] for more details). This complex region contains some other, not yet firmly identified, "hot spots".

Although the gamma-ray source spatially coincides with 
the position of Sgr $A^{*}$ (see Fig. 11), the upper limit on the angular size of the $\mathrm{TeV}$ source of a few arc minutes is still too large to exclude the link to other potential sources located within the central $\leqslant 10 \mathrm{pc}$ region. The detection of variability of the gamma-ray flux would greatly contribute to the localisation of the gamma-ray production region in Sgr A*. However, unlike the observations at radio and $\mathrm{X}$-ray wavelengths, no variability has been observed both at $\mathrm{GeV}$ and $\mathrm{TeV}$ energies. This disfavours, but still cannot discard Sgr $\mathrm{A}^{*}$ as a possible gamma-ray source, especially given that several radiation mechanism, associated with the accretion flow, are capable of explaining the reported gamma-ray fluxes [117]. Perhaps a more plausible site of gamma-ray production could be the central, dense extended region of radius of $10 \mathrm{pc}$. However, even in this scenario Sgr A* remains a potential source indirectly responsible for the gamma-ray signal through interactions of runaway particles accelerated in Sgr A*, but later injected into the surrounding dense gas environment $[118,119]$. The analysis of the combined Fermi-LAT and H.E.S.S. data show that the complex shape of the $\mathrm{GeV}-\mathrm{TeV}$ radiation can be indeed naturally explained by the propagation effects of protons interacting with the dense gas within the central $10 \mathrm{pc}$ region [115,119]. A good agreement between the data and calculations is shown in Fig. 12, where the radial profile of the gas density has been carefully taken into account. The flat spectra in the segments of the proton spectrum around $1 \mathrm{GeV}$, and at $\mathrm{TeV}$ energies (below $10 \mathrm{TeV}$ ) have different explanations. While at $\mathrm{GeV}$ energies the protons are diffusively trapped, so that they lose a large fraction of their energy before they leave the dense $3 \mathrm{pc}$ region, at $\mathrm{TeV}$ energies they propagate rectilinearly. At intermediate energies the protons start to effectively leave the inner $3 \mathrm{pc}-$ region, and the steepening of the energy spectrum can be naturally referred to the energy-dependent diffusion coefficient. What concerns the proton injection spectrum, it should be a hard power-law, close to $E^{-2}$, with an intrinsic cut-off around $100 \mathrm{TeV}$. The required total energy of protons currently trapped in the gamma-ray production region, $W_{\mathrm{p}} \simeq L_{\gamma} t_{\mathrm{pp} \rightarrow \gamma} \simeq 10^{49}\left(n / 10^{-3} \mathrm{~cm}^{3}\right)^{-1} \mathrm{erg}$ is quite modest, given that the density in the circumnuclear ring could be as large as $10^{5} \mathrm{~cm}^{-3}$ [119].

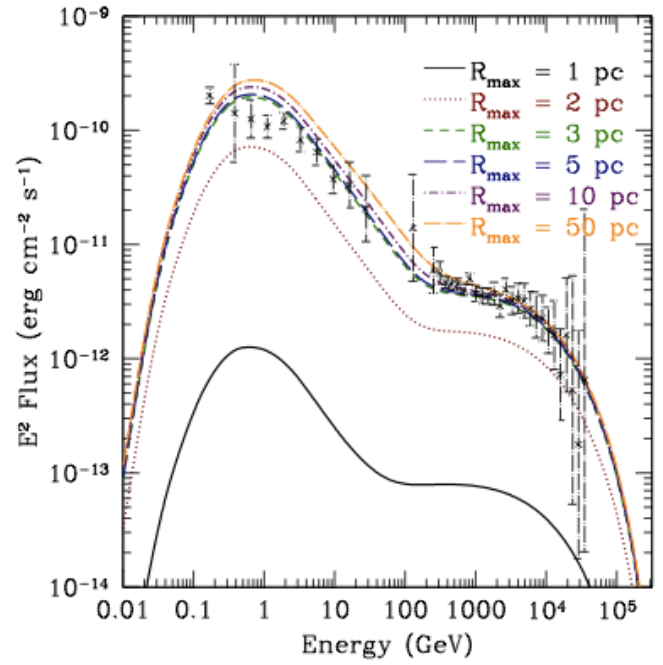

Fig. 12 Energy spectra of gamma-ray emission from GC. The Fermi-LAT [115] and H.E.S.S. data [116] are shown together with calculations of $\gamma$-rays from $p p$-interactions within radial cones of various size up to $50 \mathrm{pc}$. The flux falls off rapidly after $3 \mathrm{pc}$ because the main contribution comes from the 1.2-3 pc circum-nuclear ring. From Ref. [119], reproduced by permission of the AAS.

The interpretation of the spatially unresolved gammaray emission towards Sgr A* by interactions of runaway protons with the dense gas in the central (several pc) ring, predicts a smooth transition to another radiation component formed in more extended regions of the GC. The energy and spatial distributions of this radiation depend on the injection history of protons and the character of their diffusion. The H.E.S.S. observations of the socalled Central Molecular Zone (CMZ) of radius $\approx 200 \mathrm{pc}$ indeed revealed an extended $\mathrm{TeV}$ gamma-ray emission [122] with a clear correlation with the most prominent giant molecular clouds located in CMZ (see Fig. 11). Using the maps of $\mathrm{TeV}$ gamma-ray emission, and maps of the CS $(\mathrm{J}=1-0)$ emission which contain information about the column density in dense cores of molecular clouds, the cosmic-ray density in these clouds has been derived. It appears to be significantly enhanced (by an order of magnitude at multi-TeV energies) relative to the local cosmic-ray flux in the solar neighbourhood. This indicates to a strong non-thermal activity accompanied with proton acceleration which in the past was perhaps higher than at the present epoch. An additional support for this hypothesis comes from the spatial distribution of gamma-rays. The H.E.S.S. observations show that the ratio of gamma-ray flux to the molecular gas column density varies with galactic longitude, with a noticeable "deficit" at $l \approx 1.3^{\circ}$. This interesting feature can be interpreted as a non-uniform spatial distribution of cosmic rays, i.e. the relativistic protons accelerated in Sgr A* have not yet had time to diffuse out to the periphery of the $200 \mathrm{pc}$ region. The epoch of the high activity of the accelerator depends on the proton diffusion coefficient. 
Assuming, for example, that the propagation of multi$\mathrm{TeV}$ protons in the GC proceeds with a speed similar to the one in the Galactic Disk, the epoch of high activity of the accelerator and the total energy release in relativistic particles during the outburst are estimated to be $10^{4} \mathrm{yr}$ and $10^{50} \mathrm{erg}$, respectively [122].

High-energy processes that take place in the GC apparently play a key role in the formation of two enormous gamma-ray structures recently discovered in the Fermi-LAT data set - the Fermi bubbles [123]. Centred on the core of the Galaxy, these structures symmetrically extend to approximately $10 \mathrm{kpc}$ above the Galactic plane. The parent relativistic particles (e.g., protons) could be accelerated in the nucleus of GC, and then injected into Fermi Bubbles. Alternatively, protons and electrons could be produced in situ through firstand/or second-order Fermi acceleration mechanisms supported by hydrodynamical shocks or plasma waves in a highly turbulent medium. The processes that create and support these structures could originate either from an AGN-type activity related to the central black hole (Sgr A*) or from ongoing star formation in the galactic nucleus.

The luminosity of gamma-rays with hard, $E^{-2}$-type, spectrum in the energy interval $1-100 \mathrm{GeV}$ (see Fig. 13) is $L_{\gamma} \approx 4 \times 10^{37} \mathrm{erg} / \mathrm{s}$. Given the overall limited energy budget of the GC, particle acceleration and gamma-ray emission in the Fermi bubbles should proceed with very high efficiency. Despite the significant differences of the models proposed for the origin of the Fermi bubbles, only two radiation mechanism can be responsible for gammarays - IC emission by relativistic electrons or decays of neutral pions produced in $p p$-interactions. Because of severe radiative energy losses, however, the mean free path of $\geqslant 100 \mathrm{GeV}$ electrons is significantly shorter than the size of the Fermi bubbles. Therefore, one has to postulate in situ electron acceleration throughout the entire volume of the bubbles $[123,124]$. Such a scenario could be realised through stochastic (second-order Fermi) acceleration [125] or due to series of shocks propagating through the bubbles and accelerating relativistic electrons [126]. Importantly, the suggested acceleration mechanism seem unable to boost the electron energy beyond $1 \mathrm{TeV}$, thus in order to explain the extension of the observed gammaray spectrum up to $100 \mathrm{GeV}$ by IC, one has to invoke FIR and optical/UV background emission supplied by the galactic disk (see Fig. 13). This model provides robust predictions. In particular, since the FIR and opti$\mathrm{cal} / \mathrm{UV}$ contributions to the target field for IC scattering decrease quickly with distance from the disk, the spectrum of gamma-rays from high latitudes should contain a cut-off above tens of GeV. The limb brightening at highest energies is another characteristic feature predicted by this model. These spectral and spatial features can be explored in the near future, after the gamma-ray photon statistics in the Fermi-LAT data set has achieved an adequate level.

An hadronic origin for the observed gamma-rays is an alternative interpretation suggested for the Fermi bubbles $[124,127]$. Despite the low plasma density in the Fermi bubbles, $n \leqslant 10^{-2} \mathrm{~cm}^{-3}$, the efficiency of proton interactions can be very high. Indeed, if protons would have been continuously injected and trapped in the bubbles over timescales of approximately $10^{10} \mathrm{yr}$, the main power in accelerated protons would be lost in $p p$-collisions given that the characteristic time of the latter, $t_{\mathrm{pp}}=1 /\left(k_{p} n \sigma_{\mathrm{pp}} c\right) \approx 5 \times 10^{9}\left(n / 10^{-2} \mathrm{~cm}^{-3}\right)^{-1} \mathrm{yr}$, is shorter than the confinement time. This implies that one deals with a so-called "thick target" scenario, when the system is in saturation. The hadronic gamma-ray luminosity is equal to $L_{\gamma} \approx W_{\mathrm{p}} / t_{\mathrm{pp} \rightarrow \pi^{0}}$, where $W_{\mathrm{p}}$ is the total energy of protons in the bubbles, and $t_{\mathrm{pp} \rightarrow \pi^{0}}$ is the timescale for neutral pion production in $p p$-interactions. In the saturation regime, $W_{\mathrm{p}}=\dot{Q}_{\mathrm{p}} t_{\mathrm{pp}}$ (with $\dot{Q}_{\mathrm{p}}$ the injection rate of protons), assuming that the energy dissipation through $p p$-collisions is the dominant loss process. Since $t_{\mathrm{pp}}=1 / 3 t_{\mathrm{pp} \rightarrow \pi^{0}}$, we have $L_{\gamma}=\dot{Q}_{\mathrm{p}} / 3$, thus about a third of the power injected into relativistic CRs emerges in gamma-rays (of all energies) independent of the local density, interaction volume and the injection time. Note that since the timescale of $p p$-interactions is comparable to the supposed age of the bubbles of $10^{10} \mathrm{yr}$, the efficiency would be somewhat less. Also, one should take into account that at low energies, ionisation and adiabatic losses of protons play a non-negligible role, thus the overall efficiency for a broad energy spectrum of protons would be reduced to several percent. The fluxes of hadronic gamma-rays shown in Fig. 13 confirm these simple estimates. Note that independent of the history of injection of relativistic protons, the current total energy in protons should be as high as $W_{\mathrm{p}}=L_{\gamma} t_{\mathrm{pp} \rightarrow \pi^{0}} \simeq 10^{55}$ erg which is comparable to the magnetic field energy in the bubbles (cf. Ref. [301]).

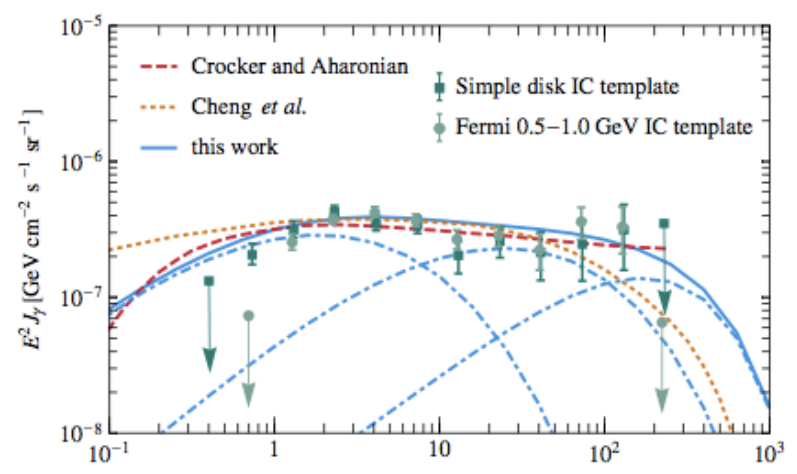

Fig. 13 The spectral energy distribution of gamma-rays from the Fermi bubbles compared to theoretical predictions. (i) IC model of Ref. [125] (solid line) assuming stochastic acceleration of electrons in the bubbles (the contributions from the scattering on the CMB, FIR, and optical/UV backgrounds are shown separately); 
(ii) IC model of Ref. [126] (dotted line) assuming diffusive shock acceleration of electrons; (iii) hadronic model of Ref. [124] (dashed line). The figure is from Ref. [125].

The above noted hadronic model of gamma-ray emission of the Fermi bubbles does not exclude other "hadronic" scenarios with faster energy release related to the activity of the central black hole Sgr A*. A fast energy release can be provided, for example, by the capture of stars by Sgr $\mathrm{A}^{*}$ over the last $10 \mathrm{Myr}$ with an average capture rate of $3 \times 10^{-5} \mathrm{yr}^{-1}$ and energy release of $3 \times 10^{52}$ erg per capture [128]. It has been argued in Ref. [140] that quasi-periodic injection of hot plasma could produce a series of strong shocks in the Fermi bubbles which can (re)accelerate protons beyond the "knee", up to energies of about $10^{18} \mathrm{eV}$. If confirmed by independent detailed hydrodynamical simulations, this could appear a viable solution for the origin of one of the most "problematic" (poorly understood) energy intervals of cosmic rays.

\subsection{Blazars}

Most of the detected extragalactic gamma-ray sources belong to the blazar class, which comprises BL Lac objects and Flat Spectrum Radio Quasars (FSRQs). The central engine in these active galaxies (AGNs), a supermassive black hole $(\mathrm{BH})$ of mass $\gtrsim 10^{7} M_{\odot}$ surrounded by an accretion disk, is commonly believed to eject a relativistic jet pointing almost directly towards the observer. Doppler boosting effects results in strong flux amplification, thus naturally favouring the detection of blazars on the extragalactic sky.

Fermi-LAT, for example, has detected over 1000 extragalactic high-energy (HE) sources in two years of survey (2LAC), most (>90\%) of which are blazars [129]. In comparison, non-blazar sources like starburst galaxies $(\mathrm{SBs})$ or radio galaxies $(R G s)$ only make out a minor fraction (in numbers).

At the time of writing more than 50 extragalactic VHE sources, populating the whole sky, are listed in the online TeV Catalog (TeVCat). I] The majority of them $(\sim 90 \%)$ are again of the blazar type, with the so-called high-frequency-peaked BL Lac objects (HBLs, with lowenergy component peaking at $\nu_{p}>10^{15} \mathrm{~Hz}$, in contrast to $L B L s=$ low-frequency-peaked BL Lacs, peaking at $\left.\nu_{p}<10^{14} \mathrm{~Hz}\right)$ constituting the dominant $(\geqslant 70 \%)$ sub-class, yet also including three FSRQs (3C279 at $z=0.536$; PKS 1510-089 at $z=0.361$ and PKS $1222+21$ at $z=0.432$ ). FSRQs are typically distinguished from BL Lac objects by the presence of strong and broad (restframe equivalent width $\geqslant 5 \AA$ ) optical emission lines. Almost all Fermi-detected FSRQs for which $\nu_{p}$ can be estimated are of the low-frequency-peaked $\left(\nu_{p}<10^{14} \mathrm{~Hz}\right)$ type. Note that AGNs, which have been detected at TeV are typically characterised by a harder $\mathrm{GeV}$ photon index than the majority of $2 \mathrm{LAC}$ sources.

At present, blazar sources out to redshift $z \sim 0.6$ (i.e., 3C279 at $z=0.536$ [163] and BL Lac KUV 00311-1938 at $z>0.51$, tentative $z=0.61$ [164]) have been detected at VHE energies, cf. Fig. 14 for their redshift distribution. Blazar population studies at lower (radio-X-ray) frequencies indicate a redshift distribution for BL Lacs objects that seems to peak at $z \sim 0.3$, with only few sources beyond $z \sim 0.8$ (under the proviso of some bias as for a substantial fraction of BL Lacs the redshift is not known), while the FSRQ population is characterised by a rather broad maximum between $z \sim(0.6-1.5)$ [160].

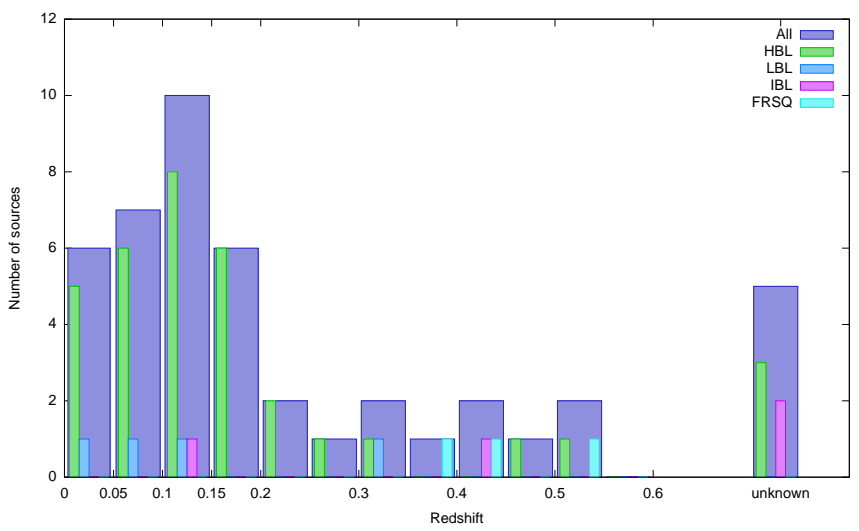

Fig. 14 Distribution of redshift for the VHE-detected blazars. Redshift data are taken from TeVCat. Most objects are within $z \lesssim 0.2$.

Observed VHE flux levels typically range from $\sim 1 \%$ (for the average/steady states) up to $\sim 15$ times the Crab Nebula steady flux in high activity stages (e.g., for the 2006 flaring state of PKS 2155-304 [137]). Gammaray emission beyond $10 \mathrm{TeV}$ has been established, with measured photon energies reaching, e.g. $\sim 20 \mathrm{TeV}$ in Mkn 501 ( $\mathrm{z}=0.034 ; 1997$ VHE flare [130]) and Mkn 421 $(z=0.031 ; 2010$ VHE flare [131]). The observed VHE spectra usually vary between hard and soft power laws; for the HBL sources, for example, inferred photon indices (assuming a single power law $d N / d E \propto E^{-\Gamma}$ ) range from $\sim 2.3$ to $\sim 4.5$, with some indications for spectral hardening with increasing activity. Non-HBL sources (i.e., $I B L=$ intermediate-peaked BL Lacs and LBLs) are usually detected during high states only, with low states expected to fall below current sensitivities.

In a $\nu F_{\nu}$ representation (SED), blazars often show a double-humped structure, cf. Figs. 15 and 16. The first hump (sometimes reaching peak frequencies up to $\sim 10^{19}$ $\mathrm{Hz}$, as in the HBL object RGB J0710+591 [132]) is commonly attributed to non-thermal electron synchrotron emission; the second one is widely believed to be due to leptonic inverse Compton processes (on the synchrotron 
photons in SSC, or on ambient photons in External Inverse Compton $[=\mathrm{EC}]$ models), although hadronic scenarios often remain possible. Different blazar population studies seem to suggest that there is a continuous spectral trend (see Fig. 15) from FSRQ $\rightarrow \mathrm{LBL} \rightarrow \mathrm{IBL} \rightarrow \mathrm{HBL}$, often called the "blazar sequence", characterised by a decreasing source luminosity, increasing synchrotron peak frequency and a decreasing ratio of high- to low-energy component [133,134] (but cf. also [135] for caveats due to selection effects and unknown redshift).

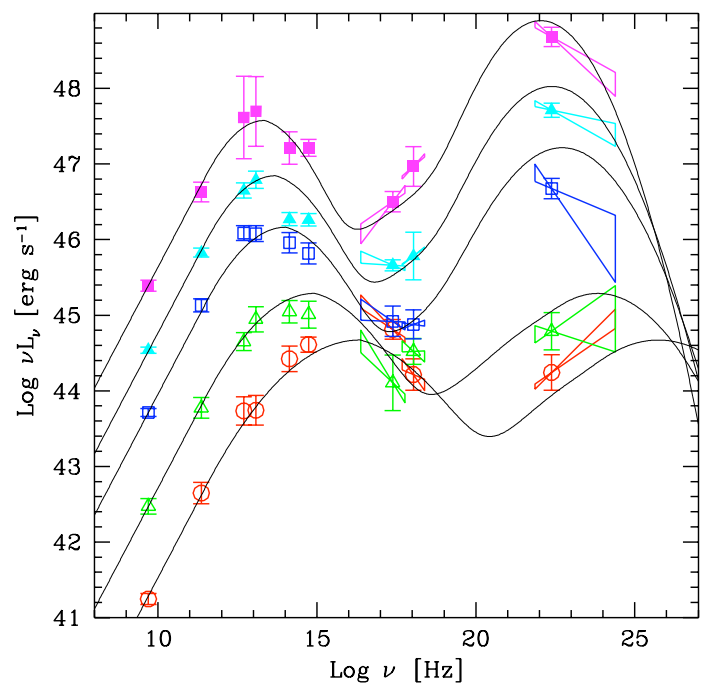

Fig. 15 Sequence of characteristic blazar SEDs as a function of source luminosity from FSRQ (top curve) to HBL objects (bottom curve). From Ref. [134], reproduced with permission (CESO.

Blazar SEDs can span almost 20 orders of magnitude in energy, making simultaneous multi-wavelength observations a particular important diagnostic tool to disentangle the underlying non-thermal processes. A variety of leptonic and hadronic emission models have been discussed in the literature (see, e.g., [136] and reference therein). A significant correlation between $\mathrm{TeV}$ and $\mathrm{X}$-ray flux variations for example, which is often found, could favour a leptonic synchrotron-Compton interpretation, but counterexamples ("orphan TeV flares") do exist [141]. Short-term variability is usually more difficult to account for in hadronic models because of longer cooling timescales, but strong magnetic fields (for proton synchrotron, e.g. [142]) or high target matter densities (pp-interactions triggered by jet-star interactions, e.g. [143]) may partly compensate. While for HBL objects, homogeneous (one-zone) leptonic SSC modeling often seems to provide a reasonable SED characterization (but see, e.g., [144] for a possible exemption), this does not apply in a similar way to LBL objects. Among the four LBLs detected, for example, AP Lib $(z=0049)$ represents an intriguing example where the 2 nd bump seems extremely broad (stretching from $\mathrm{keV}$ to $\mathrm{TeV}$ ), defying a simple homogeneous SSC interpretation [145].

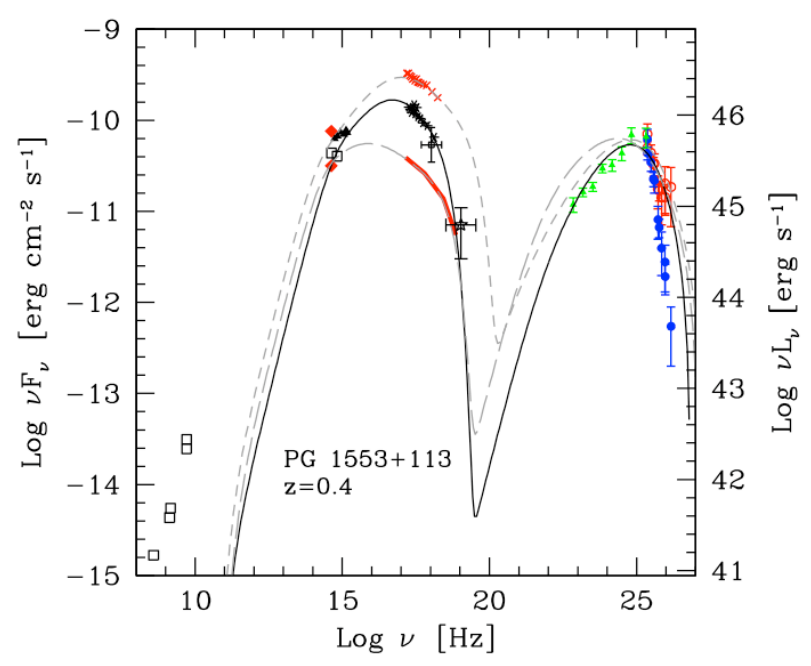

Fig. 16 A recent, double-hump-structured SED example: The high-frequency-peaked (HBL) BL Lac object PG $1553+113$ as based on VHE (MAGIC, 2005-2009) observations and archival data. Pronounced variability (on yearly time scale) is seen in the $\mathrm{X}$-ray band. The average SED has been modelled with a one-zone SSC model (continuous black line). From Ref. [159], reproduced by permission of the AAS.

\begin{tabular}{c|c|c|c} 
AGN & type & redshift & $\Delta t_{\text {VHE }}$ \\
\hline PKS 2155-304 & HBL & 0.116 & $\sim 3$ min \\
Mkn 501 & HBL & 0.034 & $\sim 3$ min \\
PKS 1222+21 & FSRQ & 0.432 & $\sim 10$ min \\
Mkn 421 & HBL & 0.031 & $\sim 10$ min \\
BL Lac & LBL & 0.069 & $\sim 15$ min \\
W Comae & IBL & 0.102 & $\sim 1$ day \\
M87 & RG & 0.004 & $\sim 1$ day
\end{tabular}

Tab. 2 VHE variability in AGN: Characteristic minimum VHE variability timescale $\Delta t_{\mathrm{VHE}}$ as observed with current instruments for an exemplary set of AGN.

Despite the limited temporal coverage of the current IACTs more than half of the AGN detected in the TeV domain shown variability, albeit often weak. For the majority of them, variability timescales above one month have been found. In about a quarter of them there is clear evidence for short-term VHE variability on observed timescales of less than one day, cf. Table 2. The HBL class currently reveals the most rapid and dramatic VHE gamma-ray flux variability with observed variability timescales $<5 \mathrm{~min}$, as found by the H.E.S.S. and MAGIC experiments for PKS 2155-304 $(z=0.116)$ [137] and Mkn 501 [138], respectively, cf. Fig. 17. Given the limited angular resolution $\left(\sim 0.1^{\circ}\right)$ of IACTs, this im- 
plies that one of the most constraining requirements on the jet kinematics and the high-energy emitting region comes from VHE variability studies. Fast VHE variability from distant blazars can also be used to derive constraints on an energy-dependent violation of Lorentz invariance (energy-dependent speed-of-light) as predicted in various models of Quantum Gravity [146,158].

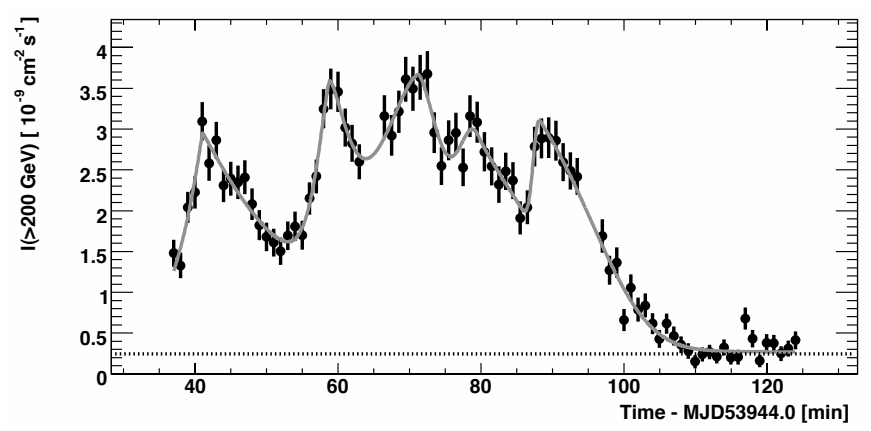

Fig. 17 Light curve: Integrated flux $I(>200 \mathrm{GeV})$ versus time as observed by H.E.S.S. for PKS 2155-304 on July 28, 2006. The data are binned in 1-minute intervals. The horizontal line gives the steady flux from the Crab Nebula for comparison. From Ref. [137].

The detection of a large number of gamma-ray emitting blazars has opened a new research area - "observational gamma-ray cosmology". The underlying idea is based on the energy-dependent absorption of $\gamma$-rays from distant extragalactic objects caused by interactions $\left(\gamma_{\mathrm{VHE}} \gamma_{\mathrm{EBL}} \rightarrow e^{+} e^{-}\right)$with the Extragalactic Background Light $(E B L)$ that extends from UV to far IR wavelengths. The identification of absorption features in the spectra of $\gamma$-rays above $10 \mathrm{GeV}$, as well as detection of characteristic angular and time distributions of gamma-rays produced during the cascade development in the intergalactic medium on large $(\geqslant 100 \mathrm{Mpc})$ scales, should allow us to derive unique cosmological information about the EBL and the intergalactic magnetic fields $(I M F s)$. The realization of these exciting possibilities requires not only precise spectroscopic measurements from a large number of extragalactic objects located at different redshifts, but, more importantly, a good understanding of the intrinsic gamma-ray spectra. So, far the most significant contribution in this area comes from the measurements of gamma-rays from blazars with redshifts between 0.1-0.2. In particular, based on such observations, the H.E.S.S. collaboration has first reported a quite meaningful upper limit on the EBL at near and mid-infrared wavelengths [147]. Remarkably, the inferred upper limit appeared to be very close to the lower limit given by the measured integrated light of resolved galaxies (galaxy counts), cf. also $[148,150,151]$ for related inferences. Very recently, a similar result has been reported by the Fermi-LAT collaboration [152] for the EBL at optical and UV bands. One should men- tion, however, that the inferred upper limits are not model-independent. The H.E.S.S. result, for example, is based on the assumption that the differential intrinsic spectrum is not harder than $E^{-1.5}$. The Fermi-LAT result is based on the detection of cutoffs in the averaged spectra of three samples of BL Lac objects combined in three different intervals of redshift, assuming that these cut-offs are caused by intergalactic absorption. Although both assumptions sound quite reasonable, and the derived upper limits agree with most of the theoretical/phenomenological predictions for the EBL, one should keep in mind that they are not free of model assumptions. It is believed that future measurements by next-generation detectors, in particular by CTA, based on a much larger sample of AGN should significantly increase the source statistics and improve the quality of data, and consequently reveal details in the EBL. This optimistic view may, however, underestimate the difficulties related to the uncertainties of the intrinsic source spectra. On the other hand, the limits in which presently the EBL fluxes are robustly constrained, are quite tight, so one can "recover" the intrinsic gammaray spectra with a reasonable accuracy. Interestingly, in the case of some blazars, the gamma-ray spectra after correction for intergalactic absorption, appear extremely hard with photon indices $\leqslant 1.5$ or even close to 1 , see e.g. $[147,150,153]$. This challenges conventional radiation models, but still cannot be considered as a failure of the standard blazar paradigm and as a need for new physics. Such spectra can still be explained, assuming, for example, the prevalence of certain conditions for the formation of the parent electron spectra (e.g., stochastic acceleration) or specific internal gamma-ray absorption, see e.g. [154-156]. Nevertheless, the growing number of VHE blazars with redshift exceeding $z \sim 0.5$ tells us that one should perhaps be prepared for even more dramatic assumptions, including violation of Lorentz invariance or "exotic" interactions involving hypothetical axion-like particles. An alternative interpretation of gamma-rays from very distant blazars (in case of their detection) exists in the framework of standard physics: TeV gammarays can in principle be observed even from a source at $z \geqslant 1$, if the observed gamma-rays are secondary photons produced in hadronic interactions (with CMB or EBL background photons) of energetic cosmic-ray protons, originating in the blazar jet and propagating over cosmological distances almost rectilinearly. In the case of a detection of $\mathrm{TeV}$ gamma-rays from a blazar with $z \geqslant 1$, this model could in principle provide a viable interpretation consistent with conventional physics, but with an extreme assumption on the strength of the IMF in the range of $10^{-17}-10^{-15} \mathrm{G}$ (see, e.g. [157]). On the other hand, if VHE $\gamma$-rays from distant blazar attenuate through pair-production with EBL photons, constraints on the strength of the IMF can be derived by model- 
ing the anticipated $\mathrm{GeV}$ emission from the electromagnetic cascades, taking the possible deflections of pairs in the IMF into account. According to a recent study, this method suggests a lower bound on the IMF of $B \gtrsim 10^{-17}$ G [149].

\subsection{Radio Galaxies}

Misaligned (non-blazar) AGNs, characterised by jets substantially inclined with respect to the observer, represent a particularly interesting class of VHE emitters. Nearby radio galaxies (RGs) are especially attractive as their proximity may allow us to resolve the radio jets down to sub-parsec scales and to study possible multiwavelength correlations. The absence of strong Doppler boosting could make a VHE detection challenging, yet also allow to get unique insights into emission regions otherwise hidden.

Out of $\sim 1000$ high-energy (HE) sources (886 in the "Clean Sample"), Fermi-LAT has reported the detection of only about ten misaligned RGs at $\mathrm{GeV}$ energies, with a predominance of the Fanaroff-Riley-type I (FR I) $[129,161,162]$. At TeV energies, only four RGs have been identified by current IACTs: The nearest AGN Cen A $(d \simeq 3.8 \mathrm{Mpc})$, the giant RG M87 ( $\simeq 16.7 \mathrm{Mpc})$, and the Perseus Cluster $(d \sim 77 \mathrm{Mpc}, z \sim 0.018)$ RGs NGC 1275 and IC 310. A detection of the RG 3C66B was initially reported by MAGIC (2007 observations [139]), but the VHE emission seems not sufficiently disentangled from the nearby (separation $\theta \sim 0.12^{\circ}$ ) IBL blazar 3C66A to include it here.

Cen $A$ was detected at VHE in a deep ( $>120 \mathrm{~h})$ exposure by H.E.S.S. with a integral flux above $250 \mathrm{GeV}$ of $\sim 0.8 \%$ of the steady flux of the Crab Nebula (corresponding to an apparent isotropic luminosity of $L(>250$ $\mathrm{GeV}) \simeq 2 \times 10^{39} \mathrm{erg} / \mathrm{s}$ ) [165]. The measured VHE spectrum extends up to $\sim 5 \mathrm{TeV}$ and is consistent with a power-law of photon index $2.7 \pm 0.5$. No significant variability has been found. Fermi-LAT has also detected $\mathrm{HE}$ emission up to $10 \mathrm{GeV}$ from the core of Cen $\mathrm{A}$, with the $\mathrm{HE}$ light curve ( $15 \mathrm{~d}$ bins) being consistent with no variability and the HE spectrum described by a comparable photon index [166]. A simple extrapolation of the Fermi HE power-law to the VHE domain, however, tends to under-predict the observed $\mathrm{TeV}$ flux. This could be indicative of an additional contribution to the VHE domain beyond the common synchrotron-Compton emission, emerging at the highest energies $[167,303]$. While the giant radio lobes are also detected at $\mathrm{GeV}$ energies (with evidence for a spatial extension beyond the radio image [168]), they are clearly excluded (given the angular resolution of H.E.S.S.) as source of the detected $\mathrm{TeV}$ emission.

The giant radio galaxy $M 87$ was the first RG detected at $\mathrm{TeV}$ energies [169]. Commonly considered as a FR I-type
RG, M87 is known to host a highly massive black hole of $M_{\mathrm{BH}} \simeq(2-6) \times 10^{9} M_{\odot}$ and to exhibit a relativistic jet misaligned by an angle $\theta \simeq(15-25)^{\circ}$, consistent with modest Doppler boosting $D=1 /\left[\Gamma_{j}(1-\beta \cos \theta)\right] \lesssim 3$ (for a recent review, see e.g. [170]). M87 has shown particularly interesting VHE features over the past years, including rapid variability (day-scale flux doubling time scales $\Delta t_{\text {obs }} \sim 1$ ) above $350 \mathrm{GeV}$ during high source states (with levels sometimes exceeding $10 \%$ of the Crab Nebula), and a hard spectrum (compatible with a power law of photons index $\Gamma \simeq 2.2$ ) extending beyond $10 \mathrm{TeV}$ [171-174]. Both the hard VHE spectrum and the observed rapid VHE variability are remarkable features for a misaligned AGN. M87 is the only RG where clear evidence for day-scale $\mathrm{TeV}$ variability (see Fig. 18) has been found, although there are now hints for day-scale variability in IC 310 as well. The VHE variations in M87 are the fastest observed in any other waveband so far, and already imply a compact size of the $\gamma$-ray emitting region $\left(R<D c \Delta t_{\mathrm{obs}} /[1+z]\right)$, comparable to the Schwarzschild radius $\left(r_{s}=(0.6-1.8) \times 10^{15} \mathrm{~cm}\right)$ of the black hole in M87. Consistent with radio evidence for the ejection of a new component from the core during a flare in 2008 [175], this could point to the black hole vicinity as the most likely origin of the variable VHE radiation.

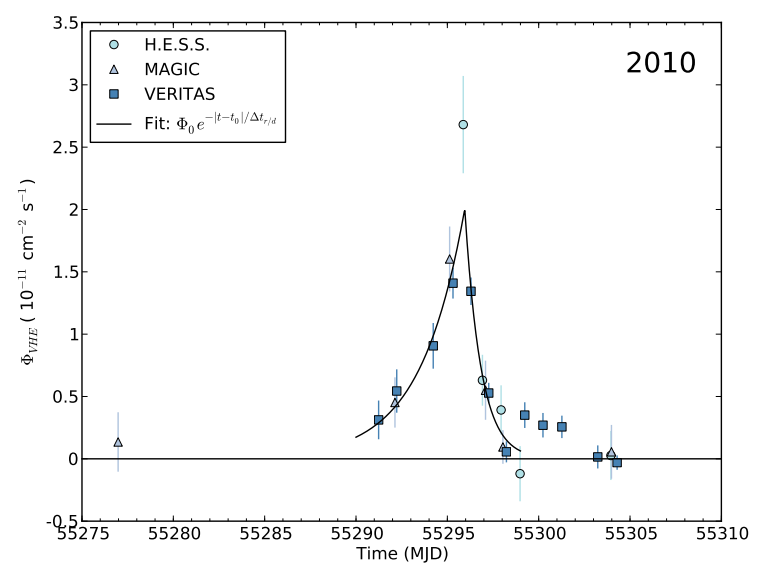

Fig. 18 VHE flare of the radio galaxy M87 as observed in April 2010 by different IACTs. Significant day-scale activity is evident. The curve shows a fit of an exponential function to the data. From Ref. [174].

IC 310 and NGC 1275 are relatively new additions, being discovered at VHE as part of a Perseus galaxy cluster campaign by MAGIC $[176,178]$. IC 310, has been originally classified as a head-tail RG. However, according to a recent high-resolution VLBA study, there are little indications for jet bending [180], rather supporting (along with the jet-to-counter-jet flux ratio) the case that IC 310 may instead rather be a weakly beamed blazar [177]. The source has been detected above $300 \mathrm{GeV}$ in about $21 \mathrm{~h}$ of data (taken in 2009/2010) at an average level of $\sim 3 \%$ of 
the Crab Nebula [176]. The VHE spectrum between 150 $\mathrm{GeV}$ and $7 \mathrm{TeV}$ is very hard (even harder than in M87) and compatible with a single power law of photon index $\Gamma \simeq 2.0$. There is clear evidence for VHE variability on yearly and monthly time scales, with indications for dayscale activity found in a new analysis, features that are all reminiscent of the VHE activity seen in M87.

On the other hand, the central dominant (FR I) cluster galaxy $N G C 1275$ (having radio jets misaligned by $\gtrsim 30^{\circ}$ ), has been recently detected above $\sim 100 \mathrm{GeV}$ during enhanced high energy (Fermi-LAT) activity in $46 \mathrm{~h}$ of data (taken between 08/2010-02/2011). While the Fermi-LAT data reveal evidence for flaring activity above $0.8 \mathrm{GeV}$ down to time scales of days [179], the situation at VHE energies is less evident. No evidence of variability has been found in the $08 / 2010$ to $02 / 2011$ VHE light curve. A recent, improved analysis of an earlier (10/2009 $-02 / 2010)$ data set, however, seems to provide hints for a possible month-type VHE variability. NGC 1275 shows a steep VHE spectrum $(\Gamma \simeq 4.1)$ extending up to $\sim 500$ $\mathrm{GeV}[178]$ and a hard HE (Fermi-LAT) spectrum (photon index $\Gamma \simeq 2.1$ ), indicative of a break or cut-off in the SED around some tens of $\mathrm{GeV}$.

\subsection{Starburst Galaxies}

Starburst Galaxies (SGs) are galaxies showing a very high rate of star formation ("starburst") in a localised region, the burst sometimes being triggered by a close encounter with another galaxy. The resultant highly increased supernova (SN) explosion rate and the expectation that the remnants (SNR) of those are efficient cosmic-ray (CR) proton accelerators (possibly up to $\sim 10^{16} \mathrm{eV}[184]$ ), suggest that starburst regions may possess a high cosmic-ray density. Because of the very high ambient gas densities $\left(n>100 \mathrm{~cm}^{-3}\right)$, hadronic interactions (inelastic proton-proton collisions and subsequent $\pi^{0}$-decay) could then lead to efficient $\gamma$-ray production, making SGs promising targets for HE and VHE astronomy.

The spiral galaxy NGC 253 is the closest $(d \sim 2.6-3.9$ Mpc) SG in the southern sky, harbouring a compact $(\sim 100 \mathrm{pc}$ in extension) starburst region with an estimated SN rate of $\sim 0.03$ year $^{-1}$. The mean density of interstellar gas in this region $\left(n \sim 600\right.$ protons $\left./ \mathrm{cm}^{3}\right)$ is almost three order of magnitude higher than the average gas density in the Milky Way. NGC 253 was detected above $220 \mathrm{GeV}$ in a deep exposure $(>119 \mathrm{~h})$ in 2005 2008 by H.E.S.S. at a flux level of $\sim 0.3 \%$ of the Crab Nebulae [181]. A recent spectral analysis of an extended (-2009) data set (Fig. 19) shows that the VHE spectrum can be described by a power law with photon index $\Gamma \simeq 2.2$, being compatible with a power-law extension of the HE (Fermi-LAT) $\gamma$-ray spectrum and little evidence for a spectral break or turnover between $200 \mathrm{MeV}$ and

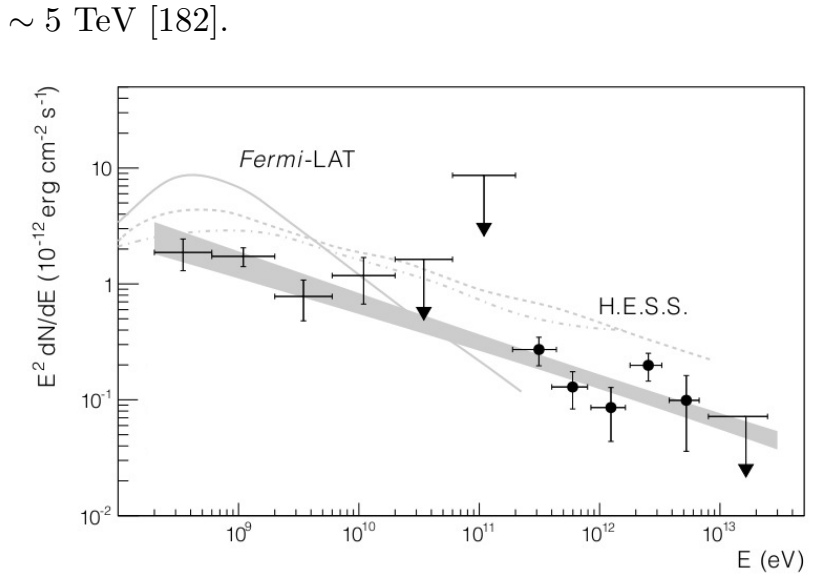

Fig. 19 Differential energy spectrum of the starburst galaxy NGC 253 from high-energy (Fermi-LAT, crosses) to the VHE (H.E.S.S., circles). The shaded area shows the $1 \sigma$ confidence band from a simultaneous fit to the high-energy and VHE data. From Ref. [182].

M82, on the other hand, is a well-known SG in the Northern Sky at a distance of $d \sim 3.9 \mathrm{Mpc}$, harbouring an active starburst region with a high SN rate of $0.1-0.3$ year $^{-1}$. This source was detected above a $700-\mathrm{GeV}$ analysis threshold in a deep exposure ( $>137 \mathrm{~h}$ ) in 2008-2009 by VERITAS at a level of $0.9 \%$ of the Crab Nebula (corresponding to $\left.L_{\gamma}(>700 \mathrm{GeV}) \simeq 2 \times 10^{39} \mathrm{erg} / \mathrm{s}\right)$ [183].

The noted SG findings are roughly compatible with hadronic CR ( $p p$-interaction) models and energyindependent (advective) particle transport from the starburst region (but see also [304,305] for an alternative approach related to the $\mathrm{TeV}$ emission from $\mathrm{PWNe}$ ). If CRs would lose most of their energy due to inelastic collisions before they escape from the starburst region ("quasi-calorimeter" scenario), the energy required in $\mathrm{TeV}$ protons would be of the order $W_{p} \simeq L_{\gamma} t_{p p} \sim$ $10^{52}\left(200 \mathrm{~cm}^{-3} / n\right) \mathrm{erg}$, with $L_{\gamma} \sim 10^{39} \mathrm{erg} / \mathrm{s}$ the characteristic VHE $\gamma$-ray luminosity, $t_{p p} \sim\left(c \sigma_{p p} k_{p} n\right)^{-1}$ the typical pp-collision time scale, and $n$ the ambient gas number density. This would imply a typical CR energy density $W_{p} / V \sim 10^{-10} \mathrm{erg} / \mathrm{cm}^{3}$, i.e., roughly two orders of magnitude higher than the average value $\left(\sim 1 \mathrm{eV} / \mathrm{cm}^{3}\right)$ for the Milky Way. Note however, that in starburst region is likely to be mildly calorimetric, with only $\sim 20-30 \%$ of the CR energy channeled into pionproduction [182].

2.9. Candidates: GRBs, Galaxy Clusters and Passive Black Holes

Several additional source classes are thought to be possible VHE emitters, but have not yet been detected. This includes GRBs, Galaxy Clusters and Passive Black Holes:

Gamma-Ray Bursts (GRBs) have been observed to emit 
intense flashes of $\gamma$-rays up to a least some tens of $\mathrm{GeV}$ energies, and their intrinsic photon spectra are likely to extend to $\mathrm{TeV}$ energies due to synchrotron-inverse Compton processes (by electrons accelerated at internal or external shocks) in leptonic models or electromagnetic cascades (triggered by $p \gamma$ - and $p p$-interactions from CR-accelerated protons) in hadronic models [185]. Extending the energy range for GRBs to the VHE domain could thus be particularly important to disentangle the origin of their high-energy emission. Significant $\gamma$-ray emission is expected to occur on time scales from second to at most hours, so that VHE instruments need to be re-posited very quickly to catch the dominant part of the outburst. The VHE prospects are, yet, generally constrained by (i) $\gamma \gamma$-pair production within the source (e.g., see the Fermi-LAT evidence for a spectral break around one $\mathrm{GeV}$ in GRB 090926A [189]), and (ii) in particular, interactions with EBL photons, limiting the detectability of VHE GRBs from cosmological distances to relatively nearby sources $(z \lesssim 0.6)$. Despite several attempts, no significant VHE excess from GRBs has been found yet and only upper limits have been reported (e.g., [186-188]). It will be highly interesting to see whether a VHE detection becomes possible in the near future with the new HESS II instrument, having a drive system designed as to reduce the response time to GRB alerts and a lower energy threshold as to reduce EBL absorption.

Massive Cluster of Galaxies, harbouring powerful AGNs and being surrounded by strong accretion shocks represent another potential VHE source class. Gamma-ray emission could be produced by several processes [190], e.g., IC up-scattering of CMB and other soft photon fields by electrons injected into the inter-cluster medium and further accelerated by turbulent processes [191,192]. Alternatively, hadronic (pp) interactions of relativistic CR protons with the ambient cluster gas could result in gamma-rays via $\pi^{0}$-decay. Given that galaxy clusters are thought to be capable of accelerating protons to energies $E>10^{18} \mathrm{eV}$ [193], $\gamma$-ray emission could also be related to IC emission by secondary electrons generated via Bethe Heitler pair production with CMB photons $\left(p+\gamma \rightarrow e^{+} e^{-}+p\right)[194,195]$. Finally, self-annihilation of a dark matter particle (e.g., WIMP) could possibly also lead to gamma-ray production [196]. However, despite these expectations, no extended gamma-ray signal has been found so far in VHE observations of local clusters. The Perseus Cluster, for example, one of the closest $(d \sim 77 \mathrm{Mpc})$ and largest clusters has been regarded as a particularly promising candidate. This cluster is characterised by intense X-ray emission and hosts a luminous radio halo (size $\sim 200 \mathrm{kpc}$ ), indicative of the presence of a high density of $\mathrm{CR}$ protons. Large intra-cluster fields $(\sim 1-10 \mu \mathrm{G})$ suggest it to be an excellent place for particle acceleration. Yet, no (extended) gamma- ray excess (but two $R G$, see above) has been found in recent VHE observations (by MAGIC for a total of $99 \mathrm{~h}$ in 10/2009-02/2011) [197]. Comparing this with simple CR model prediction suggests that the average ratio of CR-to-thermal pressure has to be below several percent. Similar (yet somewhat less stringent) CR constraints have also been inferred based on the VHE non-detection of the Coma Cluster $(d \sim 100 \mathrm{Mpc})$ by VERITAS and H.E.S.S. [198,199] (for constraints on CR mixing conditions in the Hydra Cluster, see [200]), while the VHE non-detection of the Fornax Cluster $(d \sim 19$ $\mathrm{Mpc}$ ) has been used to put constraints on the dark matter (velocity-weighted $\langle\sigma v\rangle$ ) annihilation cross-section [201] (cf. also Ref. [300] for recent $\langle\sigma v\rangle$ upper limits based on central Galactic Halo data).

Nearby quiescent or "Passive" supermassive Black Holes (PBHs), i.e., very massive black holes hosted in galactic nuclei without bright signatures of broad-band emission and with very low luminosity at lower frequency, could potentially also produce ultra-high energy cosmic rays (UHECR) and VHE gamma-rays by a number of magnetospheric processes (see [202] for review). A recent analysis [203] based on the non-detection of one of the prime candidate sources, NGC 1399 (distance 20 Mpc; black hole mass $\left.\sim 5 \times 10^{8} M_{\odot}\right)$, with current HE \& VHE instruments allows to put constraints on, e.g., gaptype particle acceleration scenarios, suggesting that this source is unlikely to be capable of accelerating protons to energies beyond $4 \times 10^{18} \mathrm{eV}$.

\section{Physics Impact of Recent Results}

\subsection{CR and Galactic Gamma-Ray Sources}

Cosmic-rays (CRs) are highly energetic protons, nuclei and electrons which are measured from $\simeq 10^{9} \mathrm{eV}$ up to $\simeq 10^{21} \mathrm{eV}$ with an almost featureless continuous spectrum. The most prominent deviation from a pure power-law (with index of about -2.8) happens in the "knee", where the index changes to $\sim-3.1$ at about $4 \mathrm{PeV}$ [204], and the "ankle", a second steepening at about $5 \times 10^{17} \mathrm{eV}$. It is widely believed that CRs below the knee are accelerated inside our Galaxy. Those CRs carry on average as much energy per unit volume as the energy density of star light, the interstellar magnetic fields, or the kinetic energy density of the interstellar gas. One hundred years after their discovery [205], the question of their origin is still open. The acceleration, accumulation and effective mixture of non-thermal particles, through their diffusion and convection in galactic magnetic fields, produce the so-called "sea" of Galactic CRs. SNRs are thought to be the main contributor to 
this CR sea. The main (phenomenological) argument in favour of this hypothesis is the CR production rate in the Galaxy, $\dot{W}_{\mathrm{CR}} \approx(0.3-1) \times 10^{41} \mathrm{erg} / \mathrm{s}$. This rate can be easily explained if one assumes that $\simeq 10 \%$ of the kinetic energy released in supernova explosions (which can reach up to $2 \times 10^{39} \mathrm{erg} \mathrm{s}^{-1} \mathrm{kpc}^{-2}$ ) is devoted to the acceleration of CRs (see, e.g., Refs. [206-208]). Moreover, this highly efficient conversion of kinetic energy of bulk motion to relativistic particles can be achieved through diffusive shock acceleration (DSA) $[7,8,184]$, which takes place in the shocks created in SNRs. A straightforward test of the acceleration of CRs in SNRs would be the detection of hadronic gamma-rays $(\mathrm{TeV} \gamma$-rays resulting from hadronic interactions through the production and decay of $\pi^{0}$-mesons) directly from young remnants [209] and/or from dense clouds overtaken by the expanding shells [210]. The DSA theory predicts observational features that can be tested with Cherenkov telescopes: The characteristic feature of shock acceleration in the non-linear regime is a concave shape of the particle energy distribution. At low $(\mathrm{GeV})$ energies, the particle spectrum is relatively steep with a differential spectral index larger than 2 , whereas it becomes very hard at the highest energies. In the presence of a strongly modified shock, the proton spectrum, just before the high-energy cut-off, can be as hard as $E^{-1.5}$ (see, e.g. [24] ). These features should then be reflected in the spectrum of secondary gamma-rays [28,40,211,212]. However, energydependent propagation effects of the particles could introduce significant modifications in the proton spectra, in particular in dense regions where most of the gammarays are produced. This applies to massive molecular clouds located outside mid-age SNRs [213,214], as well as possible, dense compact condensations inside young SNR shells [28]. Such propagation effects can lead to a substantial deviation of the observed gamma-ray spectra from the parent protons spectrum.

The new sensitive, wide-field-of-view $\mathrm{TeV}$ instruments, such as H.E.S.S., have been able to image SNRs in TeV gamma-rays, probing the SNR-shell acceleration of either electrons or hadrons up to at least $100 \mathrm{TeV}$ (in case of leptonic emission) or a few hundred $\mathrm{TeV}$ (for hadronic acceleration). The acceleration sites are spatially coincident with the sites of non-thermal X-ray emission, strengthening the hypothesis that primary Galactic CRs up to the "knee" of the energy distribution are accelerated in SNRs. However, even if radio and X-ray data suggest that SNRs are indeed the sources of CR electrons, no compelling evidence for the acceleration of protons in SNRs has been found up to now, and it is not clear whether proton and electron accelerators are of different nature. In fact, only a relatively small number of SNRs has been detected at VHE (see Sec. 2.1). A possible explanation for this is related to the evolution of SNRs, which may only be able to accelerate CRs to PeV ener- gies in the first $\sim 1000 \mathrm{yr}$, while later the high-energy hadrons escape from the system. For instance, a spectral cut-off is observed in the bright SNR RX J1713.7-3946 at $\sim 4 \mathrm{TeV}$ which implies an exponential cut-off in the proton spectrum at $\sim 25 \mathrm{TeV}$.

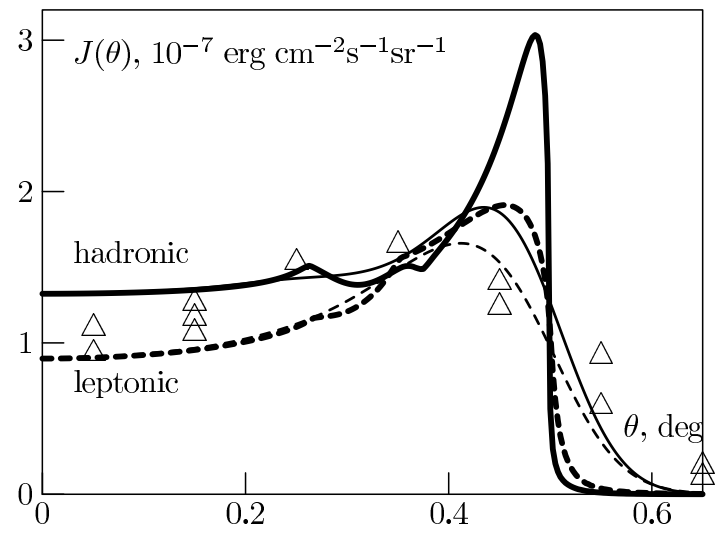

Fig. 20 Radial profiles of $1 \mathrm{TeV}$ gamma-rays calculated for the hadronic and electronic scenarios of Ref. [28] in a uniform medium (solid) and for a leptonic scenario with unmodified forward shock (dashed). The profiles, smoothed with a Gaussian point spread function with $\sigma=0.05^{\circ}$, are also shown (thin lines). The triangles correspond to the azimuthally-averaged $\mathrm{TeV}$ gamma-ray radial profile as observed by H.E.S.S. From Ref. [28].

Still, the production of gamma-rays via $p p$-interactions in dense gas condensations embedded in low-density shells represents an interesting scenario which maintains a hadronic origin as a viable option with several attractive features. The increase in photon statistic in future observations with Fermi-LAT and H.E.S.S. should help, but may hardly be sufficient to distinguish unambiguously the contributions of leptonic and hadronic interactions to the different bands of the gamma-ray spectrum. In this regard, CTA has an important role to play. This applies, first of all, to a precise measurements of the energy spectrum below $1 \mathrm{TeV}$ (down to tens of $\mathrm{GeV}$ ), and above $10 \mathrm{TeV}$ (up to $100 \mathrm{TeV}$ ). Morphological studies will provide independent and complementary information about the radiation mechanism. The low magnetic field, which is a key element in any IC model, allows multi- $\mathrm{TeV}$ electrons to propagate to large distances, and thus to fill a large volume. Because of the homogeneous distribution of target photon fields, the spatial distribution of the resulting IC gamma-rays will appear quite broad. Hadronic models, on the other hand, predict narrower and sharper spatial distributions, mainly due to the enhanced emission in the compressed region of the shock, as can be seen in Fig. 20. Using currents instruments with a typical point spread function of $\simeq 3$ arc minutes, this effect is smeared out. An angular resolution of 1-2 arc minutes, however, as reachable with CTA, would be sufficient to investigate the radial profile with enough precision to test these scenarios. 
A second univocal proof of the origin of CRs in SNRs would be the detection of $\gamma$-ray emission at extremely high energies, the so-called PeVatron $\left(1 \mathrm{PeV}=10^{15} \mathrm{eV}\right)$. In SNR shocks with relatively low acceleration rates, synchrotron losses typically prevent the acceleration of electrons to energies beyond $100 \mathrm{TeV}$. In addition, at such high energies IC emission is suppressed due to KleinNishina effects. Therefore, a contribution of IC gammarays to the radiation above $10 \mathrm{TeV}$ is expected to gradually fade out. Thus, detecting gamma-rays up to $100 \mathrm{TeV}$ would unambiguously establish a hadronic origin of the radiation.

Figure 21 shows an example for the expected X-ray and gamma-ray emission of a $T=1000$ yr-old proton PeVatron calculated for three different distributions of the accelerated protons. Both radiation components are initiated by the interactions of accelerated protons with ambient gas (of density $n=1 \mathrm{~cm}^{-3}$ ) and magnetic fields $B=300 \mu \mathrm{G}$. The gamma-ray emission arises directly from the decays of $\pi^{0}$-mesons, while the X-rays is due to the synchrotron radiation from secondary electrons (the products of $\pi^{ \pm}$-decays). The lifetime of electrons producing X-rays, $t_{\mathrm{syn}} \simeq 1.5 B_{\mathrm{mG}}^{-3 / 2}\left(E_{\mathrm{X}} / 1 \mathrm{keV}\right)^{-1 / 2} \mathrm{yr}$, is very short $(\leqslant 50 \mathrm{yr})$ compared to the age of the source. Roughly the same fraction of the energy of the parent protons is shared between the secondary electrons and the gamma-rays from $\pi^{0}$. However, since the energy of the sub-TeV electrons is not radiated away effectively, the direct $\left(\pi^{0}\right.$-decay) gamma-ray luminosity exceeds the synchrotron luminosity. The ratio $L_{\mathrm{X}} / L_{\gamma}$ depends on the proton spectrum and the injection history, and typically does not exceed 0.2-0.3. The noted calculations are based on a constant injection rate $L_{\mathrm{p}}=10^{39} \mathrm{erg} / \mathrm{s}$, giving a total energy in protons of $W_{\mathrm{p}}=L_{\mathrm{p}} \cdot T \simeq 3 \times 10^{49} \mathrm{erg}$. In order to estimate the $\mathrm{X}$-ray and gamma-ray energy fluxes (in units of $\mathrm{erg} / \mathrm{cm}^{2} \mathrm{~s}$ ) from an arbitrary PeVatron, the luminosities shown in Fig. 21 need to be multiplied with a factor $\kappa \approx 10^{-44}\left(\mathrm{nW}_{\mathrm{p}} / 3 \times\right.$ $\left.10^{49} \mathrm{erg} / \mathrm{cm}^{3}\right)(\mathrm{d} / 1 \mathrm{kpc})^{-2}$. This indicates that with the future CTA instrument, with an anticipated sensitivity at $10 \mathrm{TeV}$ of about $10^{-13} \mathrm{erg} / \mathrm{cm}^{2} \mathrm{~s}$ (cf. Fig. 1), all Galactic sources capable of accelerating particles to $\mathrm{PeV}$ energies with $\mathrm{nW}_{\mathrm{p}} \geqslant 10^{49} \mathrm{erg} / \mathrm{cm}^{3}$ should become detectable up to distances of $10 \mathrm{kpc}$.

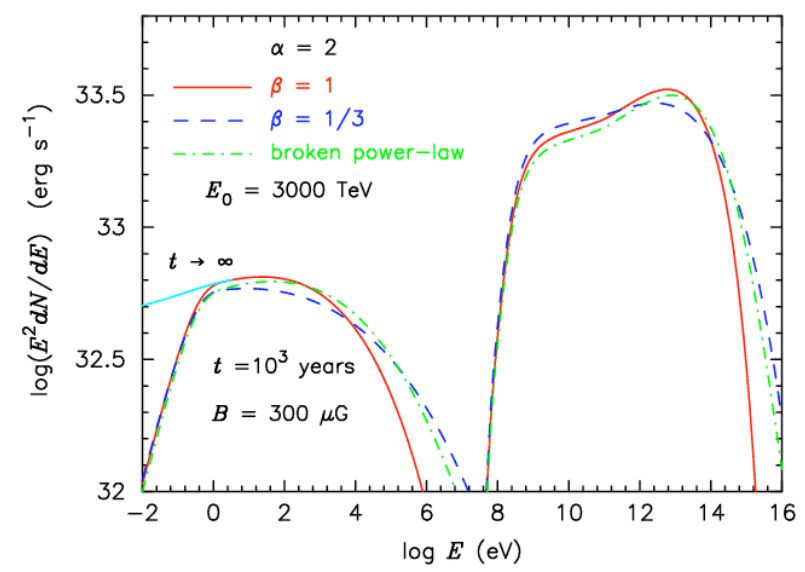

Fig. 21 Broad-band radiation of a PeVatron initiated by interactions of protons with the ambient gas. The curves are based on three different proton distributions: a single power-law with an exponential cutoff $\left(E^{-\alpha} \exp \left(-E / E_{0}\right)^{\beta}\right.$, with $\left.\alpha=2, E_{0}=3 \mathrm{PeV}\right)$ but different indices $\beta=1$ (solid curve) and $\beta=1 / 3$ (dashed curve), respectively, and a broken power-law where the spectral index changes at $E=1 \mathrm{PeV}$ from $\alpha=2$ to $\alpha=3$. From Ref. [23].

With respect to PeVatron considerations, the relevance of CR escape from the shell confinement has become more evident in the last years. Efficient acceleration and confinement of multi- $\mathrm{TeV}$ particles in SNRs can last less than several hundred years after the explosion, see e.g. [215], effectively constraining the number of SNRs emitting as PeVatrons. However, these escaping CRs could still be observable if certain conditions in the surrounding environment are fulfilled, such as the presence of massive molecular clouds which could provide a dense target to trap those running-away CRs for hadronic interactions, before they diffuse away and get integrated in the CR sea. Depending on the distance between the SNR and the molecular cloud (of typical mass of $10^{4} M_{\odot}$ ), the time of particle injection into the interstellar medium and parameters such as the diffusion coefficient, different gamma-ray spectral shapes might be expected, reaching $\mathrm{TeV}$ flux levels detectable by current instruments $[213,216]$.

Finally, the search for CRs accelerators also needs to be extended to other source classes. Different types of accelerators have been proposed to contribute to the Galactic CRs, such as superbubbles, see e.g. [217-219], or the remnants of gamma-ray bursts in our Galaxy [220]. Moreover, the CR flux measured at Earth might deviate from the Galactic one, if it would be dominated by a single nearby source (or a few local sources) in the surrounding of the solar system. The recent CR "anomalies" discovered by the PAMELA experiment, such as the very high content of positrons in their leptonic component [221], or the significant differences in the energy spectra of protons and alpha-particles [222], might be due to an oversimplification of a CR single-class-of-accelerator 
origin. In fact, a recent analysis [223] has reported variations of the CR spectrum in nearby molecular clouds embedded in the Gould Belt, which may indicate such deviation. Constraints on the CR diffusion coefficient in clouds nearby to SNRs also indicate a lower value than the one measured from the composition of the local CR spectrum (see, e.g. [224]), suggesting that the CR diffusion coefficient might not be isotropic, which could lead to differences in the $\mathrm{CR}$ spectrum in different regions of the Galaxy.

In this concern, the Galactic plane survey planned with the CTA observatory could result in the discovery of new and exciting classes of PeVatron gamma-ray sources of unknown origin [22].

\subsection{Relativistic Outflows and AGNs}

Powerful relativistic outflows and jets, with speeds approaching that of light, have been seen in a number of astrophysical sources including Active Galactic Nuclei (AGNs), Pulsars and Microquasars (MQs). If these outflows point towards us, as thought to be the case in e.g. blazars, relativistic effects can dramatically change their appearance. The dynamics of and the non-thermal processes in these outflows are important fields of active research. Some examples of current frontiers relevant to $\mathrm{TeV}$ Astronomy are mentioned below:

\subsubsection{Relativistic pulsar winds:}

Outside the light cylinder, pulsars are believed to launch highly magnetised, electron-positron winds with terminal flow speeds possibly reaching Lorentz factors up to $\Gamma_{w} \sim 10^{6}$ (for recent review see e.g., [225-227]). At the location where the interaction with the ambient medium (SNR or ISM) causes the wind to slow down, a "termination" shock is formed. It is here where efficient particle acceleration is thought to energise the particles radiating in what is observed as pulsar wind nebula (PWN). The theory of pulsar winds is usually based on the MHD description of relativistic outflows, with its electromagnetic structure being strongly dependent on the current it carries. In the simplest (force-free) approximation the plasma flow in the relativistic case becomes asymptotically radial. The magnetic field is considered frozen into the flow, so that the field structure in the far wind zone becomes almost purely azimuthal/toroidal. The pulsar wind is commonly believed to be "cold" (i.e., characterised by ordered motion only; in the co-moving frame all particle are stationary) and therefore mostly unobservable (e.g., there is no synchrotron radiation). Bulk inverse Compton scattering of ambient photons, however, could still reveal its presence ([228,229]; see also below). In the current MHD picture (cf. Fig. 22), the wind is launched as a highly magnetically (Poynting flux) dominated flow with $\sigma \equiv B^{2} / 4 \pi n m_{e} c^{2} \Gamma_{w} \gg 1$ (ratio of
Poynting to kinetic energy flux in the unshocked wind). Spectral modeling for the Crab Nebula suggests that close to its termination shock most of this energy should have been converted to the kinetic energy of the bulk motion (i.e., acceleration of the wind), resulting in $\sigma \ll 1$ [230]. How and where exactly this is achieved, however, is not yet completely understood (" $\sigma$-problem"). In an unconfined (radial) ideal MHD outflow the asymptotic Lorentz would eventually become comparable to that of the fast magnetosonic mode $\Gamma_{w, \infty} \simeq \sqrt{\sigma}$ only, implying $\sigma_{\infty} \simeq\left(\sigma_{0} \Gamma_{0}\right)^{2 / 3} \gg 1$ (e.g., typically $\sigma_{0} \sim 10^{4}, \Gamma_{0} \sim 100$ ), i.e. the wind would stay Poynting-dominated. If this were to be true, efficient first-order Fermi acceleration at the termination shock would not be possible (due to a low compression ratio and a relativistic downstream speed).

The recent detection of pulsed gamma-ray emission between $\sim(100-400) \mathrm{GeV}$ from the Crab pulsar $[57,58]$ now provides for the first time strong observational support for the presence of a (cold) ultra-relativistic pulsar wind and indicates that wind acceleration to $\Gamma_{w} \gtrsim 10^{5}$ could already occur very close to the pulsar, i.e., at distances between (20-50) light-cylinder radii) [60], see also Fig. 4. Inverse Compton up-scattering (in the KN regime) of the pulsed magnetospheric X-ray emission by pulsar wind electrons seems to be responsible for VHE emission up to $\sim \Gamma_{w} m_{e} c^{2}=500\left(\Gamma_{w} / 5 \times 10^{5}\right) \mathrm{GeV}$.

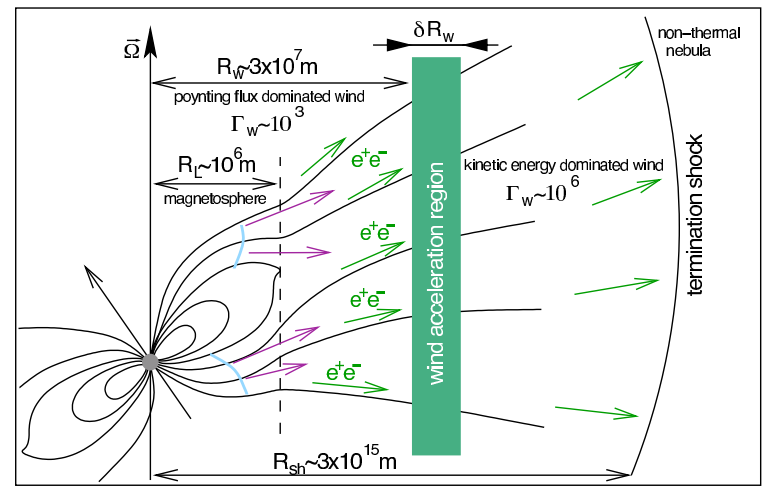

Fig. 22 Anticipated pulsar wind structure in the case of the Crab. From Ref. [60].

The apparent rapid conversion of Poynting to kinetic energy flux is certainly not easily accommodated in most of the current scenarios. It could be that this conversion attests to a longitudinal current distribution that is smaller than the Goldreich-Julian one [231]. On the other hand, if the pulsar wind would still possess a non-negligible magnetisation $(\sigma \gtrsim 0.1)$ approaching the termination shock, cross-field transport would be suppressed, making efficient operation of first-order Fermi particle acceleration unlikely. In such a case, excitation of fast growing micro-turbulence (with $\delta B / B \gg 1$ on scales $\lambda<r_{L}$ ) would be required, but seems rarely to occur under the noted conditions [232]. Magnetic field an- 
nihilation by shock-driven reconnection in a striped wind [233-235] could perhaps offer an escape route, both by producing "non-thermal" (power-law type) particle distributions in the reconnection electric field, and by generating sufficiently strong turbulence for further Fermi acceleration. Alternatively, other non-ideal MHD effects (such as conversion of the wind from a sub-luminal MHD into a superluminal electromagnetic wave, expected to be accompanied by a substantial energy transfer from fields to particles [236]) may become important in outer wind regions. Similar challenges (perhaps even more severe given the fact the here the termination shock is located much closer to the pulsar) are encountered in the gammaray binary context (cf. 3.2.3). Further VHE studies like the ones noted above may soon help us to settle a fundamental issue in current pulsar wind theory.

\subsubsection{Relativistic jets in AGNs:}

AGNs are observed to exhibit collimated (often twosided) relativistic outflows extending from sub- up to hundreds of kilo-parsecs. Radio VLBI/VLBA observations of blazar jets have revealed significant apparent superluminal motion $\left(\beta_{a}=v_{a} / c>1\right)$ of individual jet components on parsec-scale propagating away from the core. The velocity distribution of the fastest measured radio jet components peaks at $\beta_{a} \sim 10$, but seems to possess a tail extending up to $\sim 50[237,238]$. This indicates that characteristic flow speeds in AGN jets may reach bulk flow Lorentz factors up to $\Gamma \sim \beta_{a} / \beta \sim 50$. Current evidence, however, suggests that the apparent (parsec-scale) radio jet speeds in the $\mathrm{TeV}$-detected HBL sources are much slower than the speeds in the above noted, radio-selected blazars [239]. In fact, the apparent lack of superluminal speeds in the parsec-scale radio jets of TeV-HBLs seems to suggest that the bulk Lorentz factor in these object is only modest $(\Gamma \sim 2-3)$, in contrast to estimates of the (sub-parsec-scale) bulk Lorentz factors based on simple SED modelling. This could attest to the presence of some velocity gradient in the flow, such as a (longitudinally) decelerating flow $[241,242]$ or a (transversally) structured jet with a fast moving spine and a slower moving sheath [242]. If this were to be true, the radiative interplay between different zones and the possibility of further particle acceleration [243], could easily lead to complex emission patterns, thereby severely limiting classical inferences based on one-zone synchrotron-Compton modelling. Note that a spine-sheath (fast flow/slow flow)-type topology may be naturally expected within the context of current MHD models for the formation of relativistic jets. In principle, these models rely for power extraction on a source of rotation, i.e., either on the accretion disk (in a BlandfordPayne scenario) or the spin of the black hole itself (in a Blandford-Znajek scenario), see e.g. [244,245] for a recent illustration. The extent to which one of these sce- narios dominates in AGNs is not fully understood [246]. There are recent indications that the black hole spin is less important for powering AGN jets than previously believed [247], and that the radio power output in FR I type sources, believed to be the parent population of $\mathrm{BL}$ Lacs, is consistent with a rather modest spin distribution [248]. Perhaps most likely a mixture occurs in which a pair-dominated, fast-moving $(\Gamma \gtrsim 10)$ ergospheric-driven jet is encompassed by a slower-moving $(\Gamma \lesssim 10)$, diskdriven electron-proton jet. Clearly, proper modelling of the VHE source states in $\mathrm{TeV}$ blazars can shed light on these issues by constraining the required distribution of bulk flow Lorentz factors and the required power content in non-thermal particles.

The observed variability in VHE blazars with evidence for sub-hour variability (cf. Table 2) in several sources points to a compact VHE emitting region of size $R \leqslant$ $D c \Delta t_{\mathrm{VHE}}(1+z) \simeq 10^{15}(D / 10)\left(\Delta t_{\mathrm{VHE}} / 1 \mathrm{~h}\right) \mathrm{cm} . \quad$ Assuming a fiducial jet opening angle $i \sim 1 / \Gamma$, as often done, this would (without further considerations) translate into distances of $d \sim \Gamma R \ll 1 \mathrm{pc}$. While this often appears to be a good working assumption, such an inference seems severely challenged in the case of the distant VHE blazar PKS 1222+216 (4C 21.35, $z=0.432$, BH mass of a few times $\left.10^{8} M_{\odot}\right)$. PKS $1222+216$ is a bright FSRQ where the strong disk UV continuum $\left(L_{d} \sim 10^{46}\right.$ $\mathrm{erg} / \mathrm{s}$ ) is thought to photo-ionize what is called to be the "broad line region" (=BLR; often taken as a quasispherical ensemble of gas clouds moving with speeds of several $1000 \mathrm{~km} / \mathrm{s}$ at distances of $d \sim 0.1 \mathrm{pc}$ ). If the VHE gamma-rays were to originate inside the BLR, strong $\gamma \gamma$ absorption features (due to pair production in the dense BLR UV photon field) should become apparent in the observed VHE spectrum. Despite this expectation, however, the (EBL-deabsorbed) VHE spectrum as seen by MAGIC in June 2010 (coinciding with a high GeV source state) seems well compatible with a single power-law of photon index $2.7 \pm 0.3$ between $3 \mathrm{GeV}$ and $400 \mathrm{GeV}$, without evidence for an intrinsic cut-off [249], see Fig. 23. The obvious inference that the VHE emission in this source may simply originate from beyond the BLR has spurred considerable theoretical efforts and led to a rapid proliferation of models, e.g. [251-254]. One possibility is that efficient gamma-ray production occurs when the parsec-scale jet experiences a strong re-collimation shock $[250,251]$. 


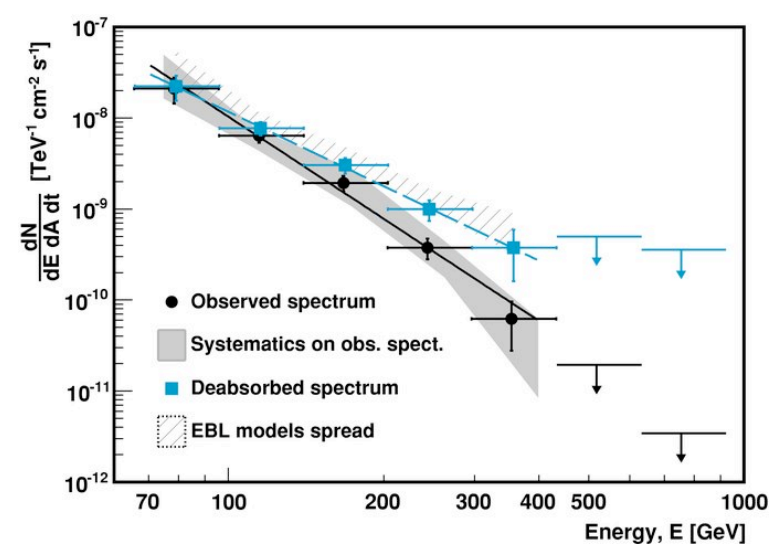

Fig. 23 Differential energy spectrum of FSRQ source PKS $1222+216$ as measured by MAGIC. Upper limits are shown as black arrows. The solid line is the best fit of the measured spectrum to a power law. From Ref. [249], reproduced by permission of the AAS.

Alternatively, the VHE gamma-rays could also result from energetic, secondary leptons produced in photohadronic $(\mathrm{n}+\gamma \rightarrow \pi)$ and $\gamma \gamma$-interactions of neutrons and UHE $\gamma$-rays with infrared photons of the more distant dust torus, provided efficient UHE proton acceleration in the inner jet, generating an escaping beam of UHE neutrons via photo-hadronic processes, and Doppler factor $D \sim 100$ do occur [252]. Other models invoke oscillations of VHE source photons into axion-like particles [293] or magnetic reconnection events [254]. What seems to be in need of further study at this stage, however, is the extent to which more a realistic BLR modelling could perhaps allow to relax some of the opacity constraints (see [255] for a first step). As the BLR is not spatially resolved on direct images, reliable information on its detailed structure and kinematics is usually not straightforwardly available. It seems likely, for example, that in reality the BLR is more complicated, e.g., [256257], being composed of multiple (geometrically different) regions (e.g., an inner, disk-ionized and an outer, jetenergized part; spherical or axial symmetric etc) and that the inferred BLR velocity is a superposition of different components (turbulence, in- and outflows, rotation etc). Interestingly, the $\mathrm{H}_{\beta}$ emission line flux in PKS 1222+216 seemed to have varied rather little during the past years (within a factor $\lesssim 1.7$ ), despite relatively large (factor $\sim 4.3$ ) optical continuum variations [259,260], which may indicate that the overall BLR is not strongly affected by the observed variable and supposedly highly beamed jet emission. Optical and VHE studies along this line could obviously help to shed more light on the BLR structure in powerful bazars.

For M87 (being more massive than PKS $1222+216$ by an order of magnitude) the observed day-scale VHE activity is already comparable to the light travel time across Schwarzschild-radius distances. Under the condi- tion of moderate Doppler boosting ( $D \lesssim 3$, as expected for a misaligned jet-source) the observed variable and hard VHE emission should originate from near to the black hole. This is facilitated by the fact that M87 is highly under-luminous (compared to Eddington, and in contrast to PKS 1222+216), making it possible for VHE $\gamma$-rays to escape from its inner jet environment. A multitude of VHE emission scenarios, relying on either hadronic and/or leptonic radiation processes, have been developed and applied within recent years to get insight into the real nature of the source (see [170] for review and references). In the context of leptonic models, one interesting variant assumes relativistic (Petschek-type) reconnection as acceleration mechanism of electrons to high energy and external Compton scattering to account for the VHE emission [261,262]. Efficient reconnection could possibly lead to an additional relativistic velocity component of the ejected plasma (in the bulk jet frame), making differential Doppler boosting possible, thereby circumventing the problem of a modest Doppler factor for the general bulk flow. It is, however, not clear at the moment whether the required conditions (e.g., high magnetisation for an electron-proton jet, negligible guide field) are likely to prevail in AGN jet. Within hadronic models, on the other hand, one recent development utilises VHE gamma-ray production via inelastic proton-proton (pp) collisions, assuming that interactions of a red giant star or a massive, dense gas cloud with the base of the jet could occasionally introduce the required high amount of target matter [263]. Recent modelling suggests that such a scenario could possibly account for the observed temporal and spectral properties if the jet is powerful enough and a sufficiently large fraction of it could be channeled into VHE gamma-ray production. An alternative explanation has been pursued in the context of magnetospheric models (see [202] for review and references), motivated by the observed day-scale VHE variability (comparable to Schwarzschild scales) and the observed radio-VHE connection in 2008 [175]. In a recent scenario [264], pair creation in a hot accretion flow (ADAF) is considered to lead to the injection of primary electrons and positrons into magnetospheric vacuum gaps, in which they are quickly accelerated to very high Lorentz factors. Curvature emission and, in particular, Compton up-scattering of (ADAF) disk photons then results in $\gamma$-rays with a spectrum extending up to $10^{4} \mathrm{TeV}$. For low accretion rates, photons with energies below several $\mathrm{TeV}$ could escape $\gamma \gamma$-absorption. On the other hand, IC photons with energies above $\sim 10 \mathrm{TeV}$ could interact with the ambient radiation field initiating pair cascades just above the gap. This would lead to a large pair multiplicity and ensures that a force-free outflow becomes established, accounting for the jet feature as seen in radio VLBA.

Obviously, due to its proximity and low luminosity, M87 
seems to bear the rare potential to give us important insights into the near black hole environment of active galaxies. Future, more sensitive VHE observations will be important to study the spectral variability and further constrain the time scales of the VHE flux variations, while simultaneous radio observations will have the potential to pin down the location of the VHE emission on spatial scales $\lesssim 10^{2} r_{s}$.

Perhaps the most prominent example for extreme shorttime VHE variability is the BL Lac object PKS 2155304. In July 2006, clear evidence for minute-timescale $\left(t_{v} \sim 200 \mathrm{sec}\right)$ VHE variability was found during a dramatic outburst (with flux level varying between 1 and 15 Crab units) [137]. The remarkable data set made a detailed temporal characterisation of the flaring period possible, showing that the variability power is distributed over (temporal) frequencies according to a red-noise-type PSD (power spectra density) $\propto \nu^{-\beta}$, with $\beta \simeq 2$ in the frequency range $\left(10^{-4}-10^{-2}\right) \mathrm{Hz}$, see Fig. $24[137,265]$ (cf. also [266] for a related PSD GeV variability analysis below $\sim 10^{-6} \mathrm{~Hz}$ ).

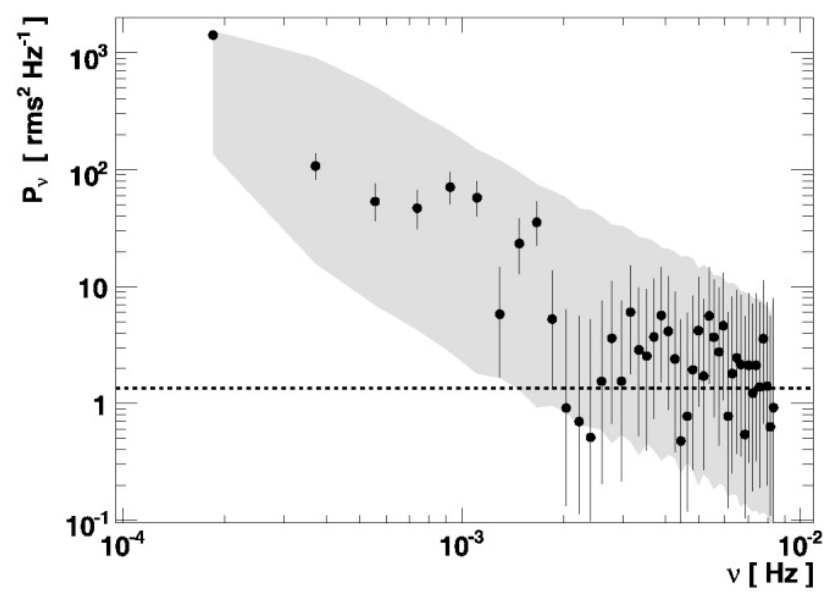

Fig. 24 Power spectrum density (PSD) of the light curve of PKS 2155-304. The grey shaded area corresponds to the $90 \%$ confidence interval for a light curve with a PSD $\propto \nu^{-\beta}$. The dotted horizontal line shows the average noise level. From Ref. [137].

Similarly to (previous) results in the X-ray domain, a correlation between (absolute) rms (root mean square) variability amplitude and mean VHE flux has been found. This is known to be characteristic of a non-linear, log-normal stochastic process and suggests that the underlying process driving the VHE variability is of multiplicative (not additive) nature (see [267], but cf. [268] for a possible caveat). The extreme short-term variability has attracted considerable attention, with a number of interesting models developed to account for it (e.g., spine-sheath [269], reconnection/ mini-jets [270], jet-star interactions [271], non-ideal MHD effects [272] or turbulence [273]). Like in Mkn 501, the VHE variability in PKS 2155-304 seems to occur on timescales much shorter than the horizon light-crossing time for a typical black hole mass inferred from its host galaxy luminosity. This could suggest that the variability originates in a small region within a highly relativistic outflow having bulk Lorentz factors $\sim 50$ [274] (but see [275] for an interesting alternative based on time-dependent modelling). The detailed temporal characteristics of the flare (PSD and log-normality) have not yet received as much attention. This situation needs to be remedied as it could offer the potential to distinguish between the different models proposed. Linear RMS-flux relations, for example, are known from black hole X-ray binaries and believed to be an essential property of the accretion flow around black holes [276], likely produced by small, independent fluctuations in the accretion rate [277]. An application of such a scenario to PKS 2155-204 is possible, but seems to require the presence of a supermassive binary black hole system [278]. The increased sensitivity of future instruments like CTA will allow us to probe the temporal characteristics of flaring $\mathrm{TeV}$ blazars beyond the usual minimum variability considerations and thereby offer an important diagnostic tool to uncover the real nature of the source.

\subsubsection{Relativistic outflows in galactic VHE binaries:}

Galactic VHE binaries are composed of a compact object (a stellar mass black hole in accreting binary systems=Microquasar $[\mathrm{MQ}]$ scenario as in e.g., Cygnus X1 ; or a ms-pulsar in pulsar wind binaries as in PSR B1259-63) and a bright companion star orbiting around each other. Complex interactions between the stellar wind and the outflow produced by the compact object (a mildly relativistic MQ jet, or an ultra-relativistic pulsar wind) are naturally expected to occur, potentially triggering efficient dissipation and gamma-ray production through a variety of processes (see [279] for review). Current research frontiers in this context include the following:

(1) The interactions of a relativistic pulsar wind with the wind from a stellar companion can result in the formation of an extended cometary-shaped radio structure rotating with the orbital period [280]. Electrons are thought to be efficiently energised in the termination shock region, producing HE and VHE gamma-rays by inverse Compton up-scattering of the stellar UV photons whilst being advected away [280,281]. In the case of the pulsar wind binary PSR B1259-63, observational evidence for variable and extended (AU-scale) radio emission has been found, demonstrating that extended (jet-like) structures can be produced in pulsar wind binaries [282]. Rotating jet-like radio features have also been detected in LS 5039 and LS I +61 303 [283-285], whose natures are not yet established. In analogy to PSR B1259-63, these findings could suggest a pulsar wind binary interpretation. On the other hand, jet-stellar outflow interactions may be 
capable of producing similar features [286,288], and MQ jet precession (with variable Doppler boosting) has been recently favoured for LS 5039 based on the finding that its radio emission displays double-sided structures changing to one-sided ones [284]. The situation could be even more complicated in that the extended radio emission may partly also be related to synchrotron emission from secondary pairs generated in $\gamma \gamma$-interactions [287]. What seems to be an open issue for a pulsar wind interpretation when applied to LS I +61 303 is that for a reasonable $\gamma$-ray production efficiency the required $\mathrm{PW}$ momentum flux significantly exceeds the stellar (Be) wind one, making the formation of elongated cometary-shaped radio structures unlikely [288].

(2) The location of the VHE emitting region is not yet understood and different (not mutually exclusive) emitting sites are conceivable (see also above). In principle, VHE gamma-rays could be produced inside the binary (pulsar wind zone or jet base), on binary scales (wind collision/interaction region) or on even larger scales (jet termination). In the case of LS 5039, the VHE data favour an origin of the $\mathrm{TeV}$ emission on binary $\left(\sim 10^{12}\right.$ $\mathrm{cm})$ scales $[90,289]$. The possibility of efficient particle acceleration (at shocks) then becomes an open issue. If particle acceleration occurs inside a MQ jet, only mildly relativistic flow speeds may be expected. If, on the other hand, particle acceleration is believed to occur in the shock region formed by the encounter of the pulsar wind and the stellar wind (standoff distance much closer to the pulsar compared to the termination shock for isolated pulsars), efficient first-order Fermi acceleration may be difficult to achieve (cf. section 3.2.1.). A comparison of the HE and VHE emission in PSR B1259-63 indicates that the spectacular $\mathrm{GeV}$ flare seen by Fermi is not accompanied by a similar flare in the $\mathrm{TeV}$ regime, suggesting that the $\mathrm{GeV}$ and the $\mathrm{TeV}$ emission are produced by different mechanisms [290]. Similarly, in the case of LS 5039 and LS I +61 303, the exponential cut-off at a few $\mathrm{GeV}$, combined with the hard spectrum at $\mathrm{TeV}$ and the anti-correlation between the two bands, points towards different origins for their $\mathrm{GeV}$ and $\mathrm{TeV}$ emissions [291]. Detailed numerical simulations of stellar/pulsar wind or stellar wind/MQ jet interactions on different scales, see e.g. [292-294], are expected to improve our understanding of the dominant VHE emitting region.

(3) Finally, interactions of the jet with the ambient medium/ISM (termination) may also result in efficient particle acceleration facilitated by, e.g., re-collimation, forward or reverse shocks, and possibly lead to VHE gamma-ray production that may eventually become detectable [295]. If so, then this could allow to more tightly constrain the jet kinetic power and the magnetic field in the interaction region.

While further high-resolution radio observations bear the promise to reveal the true origin of the changing radio morphology in gamma-ray binaries, high(er) sensitivity VHE observations with dense temporal coverage are expected to shed new light on the non-thermal mechanisms responsible for $\mathrm{TeV}$ gamma-ray production, and will eventually help to disclose the real nature of these sources.

\section{Conclusion and Perspectives}

The remarkable achievements of observational gammaray astronomy over the last decade, and the recent theoretical and phenomenological studies of acceleration and radiation processes in astrophysical environments, fully justify the further exploration of the sky at high and very-high energies. Although generally the main motivations of gamma-ray astronomy remain unchanged, the recent observational results have revealed new features which in many cases require revisions of existing theoretical models and formulations of new concepts. It is expected that over the next decade the ongoing operation of Fermi will be accompanied by observations with the current (H.E.S.S., MAGIC, VERITAS) and planned (CTA, HAWC) ground-based detectors. The data obtained in the enormous energy range from $100 \mathrm{MeV}$ to $1 \mathrm{PeV}$ will provide very deep insight into a number of problems of high-energy astrophysics and fundamental physics.

Acknowledgements FMR acknowledges LEA support, and EOW support by a RYC fellowship.

\section{References}

[1] F. Aharonian, J. Buckley, T. Kifune and G. Sinnis, Reports on Progress in Physics, 2008, 71: 096901

[2] J.A. Hinton and W. Hofmann, ARA\&A, 2009, 47: 523

[3] A.U. Abeysekara et al. (HAWC Collaboration), APh, 2012, 35: 641

[4] M. Actis et al. (CTA consortium), Experimental Astronomy, 2011, 32: 193

[5] A.V. Plyasheshnikov, F.A. Aharonian and H.J. Völk, JPhG, 2000, 26: 183

[6] F.A. Aharonian et al., APh, 2001, 15: 335

[7] L.O'C. Drury, RPPh, 1983, 46: 973

[8] R. Blandford and D. Eichler, Physics Reports, 1987, 1154: 1

[9] J. Albert et al. (MAGIC Collaboration), A\&A, 2007, 747: 937

[10] V.A. Acciari et al. (VERITAS Collaboration), ApJ, 2010, 714: 163

[11] F.A. Aharonian et al. (HEGRA Collaboration), A\&A, 2001, 370: 112

[12] V.A. Acciari et al. (VERITAS Collaboration), ApJ, 2011, 730: L20 
[13] F. Acero et al. (H.E.S.S. Collaboration), A\&A, 2010, 516: A62

[14] R. Enomoto et al. (Cangaroo Collaboration), Nature, 2002, 416: 823

[15] F.A. Aharonian et al. (H.E.S.S. Collaboration), Nature, 2004, 432: 75

[16] F.A. Aharonian et al. (H.E.S.S. Collaboration), A\&A, 2005, 437: L7

[17] F.A. Aharonian et al. (H.E.S.S. Collaboration), ApJ, 2009, 692: 1500

[18] A. Abramowski et al. (H.E.S.S. Collaboration), A\&A, 2011, 531: A81

[19] W.W. Tian et al., ApJ, 2010, 712: 790

[20] W.W. Tian et al., ApJ, 2008, 679: L85

[21] F.A. Aharonian et al. (H.E.S.S. Collaboration), A\&A, 2008, 484: 435

[22] F. Acero et al. (for the CTA Collaboration), APh, 2012, to appear (arXiv:1209.0582)

[23] F.A. Aharonian, Very high energy cosmic gamma radiation : a crucial window on the extreme Universe, World Scientific Publishing, 2004

[24] M.A. Malkov and L. O'C Drury, Reports of Progress in Physics, 2001, 64: 429

[25] Y. Uchiyama et al., Nature, 2007, 449: 576

[26] J. Vink and M. Laming, ApJ, 2003, 584: 758

[27] V.N. Zirakashvili and F.A. Aharonian, A\&A, 2007, 465: 695

[28] V.N. Zirakashvili and F.A. Aharonian, ApJ, 2010, 708: 965

[29] T. Tanaka et al. (Fermi Collaboration), ApJ, 2011, 740: L51

[30] F. Giordano et al. (Fermi Collaboration), ApJ, 2012, 744: L2

[31] A.A. Abdo et al. (Fermi Collaboration), ApJ, 2011, 734: 28.

[32] A.A. Abdo et al. (Fermi Collaboration), ApJ, 2010, 710: L2.

[33] T. Inoue et al., ApJ, 2012, 744: 71

[34] Y. Uchiyama et al.,ApJ, 2010, 723: L122

[35] A.M. Atoyan et al.,A\&A, 2000, 355: 211

[36] A.M. Atoyan and C. Dermer, ApJ, 2012, 749: L26

[37] H. Völk et al., A\&A, 2008, 483: 529

[38] T. Tanaka et al., ApJ, 2008, 685: 988

[39] B. Katz and E. Waxman, JCAP, 2008, 1: 18

[40] D.C. Ellison et al., ApJ, 2010, 712: 287

[41] J. Aleksić et al. (MAGIC Collaboration), A\&A, 2012, 541: 11

[42] A. Fiasson et al. (H.E.S.S. Collaboration), Proceedings of the 31st ICRC, Ĺodz 2009.

[43] F.A. Aharonian et al. (H.E.S.S. Collaboration), A\&A, 2008, 481: 401

[44] J. Albert et al. (MAGIC Collaboration), A\&A, 2007, 664: L87

[45] V.A. Acciari et al. (VERITAS Collaboration), ApJ, 2009,
698: L113

[46] M. Tavani et al. (AGILE Collaboration), ApJ, 2010, 710: L151

[47] A.A. Abdo et al. (Fermi Collaboration), ApJ, 2010, 712: 459

[48] A.A. Abdo et al. (Fermi Collaboration), ApJS, 2010, 187: 460

[49] A.G. Muslimov and A.K. Harding, ApJ, 2003, 588: 430

[50] A.G. Muslimov and A.K. Harding, ApJ, 2004, 606: 1143

[51] K.S. Cheng et al. ApJ, 2000, 537: 965

[52] S.S. Komissarov, MNRAS, 2002, 336: 759

[53] J.G. Kirk, Phys. Rev. Lett., 2004, 92:18

[54] R. Bühler et al. ApJ, 2012, 749: 26

[55] A.A. Abdo et al. (Fermi Collaboration), ApJ, 2010, 708: 1254

[56] E. Aliu et al. (MAGIC Collaboration), Science, 2008, 322: 1221

[57] J. Aleksic et al. (MAGIC Collaboration), A\&A, 2012: 540, 69

[58] E. Aliu et al. (VERITAS Collaboration), Science, 2011, 334: 69

[59] J. Takata et al., MNRAS, 2006, 366:1310

[60] F.A. Aharonian, S. Bogovalov, D. Khangulyan, Nature, 2012: 482,507

[61] E. de Oña Wilhelmi et al. (CTA Collaboration), APh, 2012, in press (arXiv:1209.0357)

[62] B.M. Gaensler and P.O. Slane, ARA\&A, 2006, 44: 17

[63] A. Abramowski et al. (H.E.S.S. Collaboration), A\&A, 2012, 545: L2

[64] F.A. Aharonian et al. (HEGRA Collaboration), ApJ, 2004 641: 897

[65] T.C. Weekes et al., ApJ, 1989, 342: 379

[66] C.F. Kennel and F.V. Coroniti, ApJ, 1984, 283: 694

[67] S.V. Bogovalov et al., MNRAS, 2005, 358: 705

[68] D. Volpi et al., A\&A, 2008, 485: 337

[69] J. Albert et al. (MAGIC Collaboration), ApJ, 2008, 674: 1037

[70] T. Tanimori et al. (CANGAROO Collaboration), ApJ, 1998, 497: L25

[71] F.A. Aharonian et al. (H.E.S.S. Collaboration), ApJ, 2006, 457: 899

[72] M. Tavani et al. (AGILE Collaboration), Science, 2011, 331: 736

[73] S.S. Komissarov and Y.E. Lyubarsky, MNRAS, 2004, 349: 779

[74] M. Lyutikov et al., MNRAS, 2012, 2769:

[75] W. Bednarek and W. Idec, MNRAS, 2011, 414: 2229

[76] A.P. Lobanov et al., A\&A, 2011, 533: A10

[77] Q. Yuan et al. ApJ, 2011, 730: L15

[78] F.A. Aharonian et al., MNRAS, 1997, 291: 162 
[79] J. Albert et al. (MAGIC Collaboration), ApJ, 2008, 675: L28

[80] F.A. Aharonian et al. (H.E.S.S. Collaboration), A\&A, 2005, 435: L17

[81] F.A. Aharonian et al. (H.E.S.S. Collaboration), A\&A, 2012, in press

[82] F.A. Aharonian et al. (H.E.S.S. Collaboration), A\&A, 2006, 460: 365

[83] F.A. Aharonian et al. (H.E.S.S. Collaboration), A\&A, 2012, in press

[84] J.M. Blondin et al., ApJ, 2001, 563: 806

[85] F.A. Aharonian et al. (H.E.S.S. Collaboration), A\&A, 2005, 432: L25

[86] A. Djannati-Atai et al. (H.E.S.S. Collaboration), Proc. 30th ICRC, Merida 2007

[87] F. Acero et al. (H.E.S.S. Collaboration), Proc. 32nd ICRC, Beijing 2011 (arXiv:1201.0481)

[88] P. Hofverberg et al. (H.E.S.S. Collaboration), Proc. 32nd ICRC, Beijing 2011 (arXiv:1112.2901)

[89] W. Bednarek, MNRAS, 2006, 368: 579

[90] D. Khangulyan, F. Aharonian and V. Bosch-Ramon, MNRAS, 2008, 383: 467

[91] A. Sierpowska-Bartosik and D.F. Torres, ApJ, 2008, 30: 239

[92] G. Dubus, A\&A, 2006, 451: 9

[93] D. Khangulyan et al., IJMPD, 2008, 17: 1909

[94] M. Tavani and J. Arons, ApJ, 1997, 477: 439

[95] J.G. Kirk et al., APh, 1999, 10: 31

[96] F.A. Aharonian et al. (H.E.S.S. Collaboration), A\&A, 2005, 442: 1

[97] G. Maier et al. (VERITAS and H.E.S.S. Collaboration), Proc. 32nd ICRC, Beijing 2011 (arXiv:1111.2155)

[98] J. Aleksic et al. (MAGIC Collaboration), ApJ, 2012, 754L: $10 \mathrm{~A}$

[99] F.A. Aharonian et al. (H.E.S.S. Collaboration), Science, 2005, 309: 746

[100] J. Albert et al. (MAGIC Collaboration), Science, 2006, 312: 1771

[101] J. Albert et al. (MAGIC Collaboration), ApJ2009, 693: 1

[102] V.A. Acciari et al. (VERITAS Collaboration), ApJ, 2008, 679, 2: 1427

[103] A. Abramowski et al. (H.E.S.S. Collaboration), A\&A, 2012, 541: A5.

[104] J. Albert et al. (MAGIC Collaboration), ApJ, 2007, 665: L51

[105] A.A. Abdo et al. (Fermi Collaboration), ApJ2011, 736: L11

[106] P.H.T. Tam et al., ApJ, 2011, 736: L10

[107] D. Khangulyan et al. ApJ, 2012, 752: L17

[108] S. D. Bongiorno et al., ApJ, 2011, 736: L11
[109] P.C. Gregory, ApJ, 2002, 525: 427

[110] A.A. Zdziarski et al., MNRAS, 2010, 403: 1873

[111] V.A. Acciari et al. (VERITAS Collaboration), ApJ, 2011, 738: 3

[112] R. A. Ong (VERITAS Collaboration), AN, 2011, 3153

[113] D. Hadasch et al. (Fermi Collaboration), ApJ, 2012, 749: 1

[114] M.J. Coe et al. (Fermi Collaboration) Science, 2012, 335 6065

[115] M. Chernyakova et al., ApJ, 2011, 726: 60

[116] F. Aharonian et al. (H.E.S.S. Collaboration), A\&A, 2009, 503: 817

[117] F. Aharonian and A. Neronov, ApJ, 2005, 619: 306

[118] F. Aharonian and A. Neronov, Ap\&SS, 2005, 300: 255

[119] T. Linden, E. Lovegrove and S. Profumo, ApJ, 2012, 753: 41

[120] F. Aharonian et al. (H.E.S.S. Collaboration), A\&A, 2004, 425: L13

[121] F. Acero et al. (H.E.S.S. Collaboration), MNRAS, 2010, 402: 1877

[122] F. Aharonian et al. (H.E.S.S. Collaboration), Nature, 2006, 439: 365

[123] M. Su, T.R. Slatyer and D.P. Finkbeiner, ApJ, 2010, 724: 1044

[124] R.M. Crocker and F. Aharonian, Phys. Rev. Lett., 2011, 106: 101102

[125] P. Mertsch and S. Sarkar, Phys. Rev. Lett., 2011, 107: 091101

[126] K.S. Cheng et al., ApJ, 2011, 731: L17

[127] C. Lunardini and S. Razzaque, Phys. Rev. Lett., 2012, 108: 221102

[128] K.S. Cheng et al., ApJ, 2012m 746: 116

[129] M. Ackermann et al. (Fermi-LAT Collaboration), ApJ 2011, 743: 171

[130] F. Aharonian et al., A\&A, 1999, 349: 11

[131] M. Tluczykont et al. (for the H.E.S.S. Collaboration), POS(Texas2010) 2010, 197

[132] V. Acciari et al., ApJ, 2010, 715: L49

[133] G. Fossati et al., MNRAS, 1998: 299, 433

[134] D. Donato et al., A\&A, 2001: 375, 739

[135] P. Giommi et al., MNRAS, 2012: 420, 2899

[136] M. Böttcher, in: Fermi meets Jansky, 2010, arXiv: 1006.5048

[137] F. Aharonian et al. (H.E.S.S. Collaboration), ApJ, 2007 , 664: L71

[138] J. Albert et al. (MAGIC Collaboration), ApJ, 2007, 664: 71

[139] E. Aliu et al. (MAGIC Collaboration), ApJL, 2009, 692: L29

[140] J. Aleksic et al. (MAGIC Collaboration), ApJ, 2010, 710: 
634

[141] H. Krawczynski et al., ApJ, 2004, 601: 151

[142] F.A. Aharonian, NewA, 2000, 5: 377

[143] M. Barkov et al., ApJ, 2012, 749: 119

[144] E. Aliu et al. (VERITAS Collaboration), ApJ, 2012, 750: 94

[145] P. Fortin et al. (for the H.E.S.S. Collaboration), Proc. of the 25th Texas Symposium on Relativistic Astrophysics, PoS(Texas2010), 199

[146] A. Abramowski et al. (H.E.S.S. Collaboration), APh, 2011, 34: 738

[147] F. Aharonian et al. (H.E.S.S. Collaboration), Nature, 2006, 440: 1018

[148] J. Albert et al. (MAGIC Collaboration), Science, 2008, 320: 1752

[149] A.M. Taylor, I. Vovk and A. Neronov, A\&A, 2011, 529: 144

[150] F. Aharonian et al. (H.E.S.S. Collaboration), A\&A, 2007, 475: L9

[151] M. Meyer, M. Raue and D. Horns, A\&A, 2012, 542: 59

[152] M. Ackermann et al. (Fermi-LAT Collaboration), Science, 2012, DOI: $10.1126 /$ science 1227160

[153] A. Neronov, D. Semikoz and A.M. Taylor, A\&A, 2012, 514: 31

[154] K. Katarzynski et al., MNRAS, 2007, 368: L52

[155] E. Lefa, F.M. Rieger and F.A. Aharonian, ApJ, 2011, 740: 64

[156] O. Zacharopoulou et al., MNRAS, 2011, 738: 157

[157] F. Aharonian et al., Preprint 2012, arXiv:1206.6715

[158] J. Albert et al. (MAGIC Collaboration), Phys. Lett. B, 2008, 668: 253

[159] J. Aleksic et al. (MAGIC Collaboration), ApJ, 2012, 748: 46

[160] E. Massaro et al., A\&A, 2009, 495: 691

[161] P.L. Nolan et al. (Fermi-LAT Collaboration), ApJS, 2012, 199: 31

[162] A. Abdo et al. (Fermi-LAT Collaboration), ApJ, 2010, 720: 912

[163] J. Albert et al. (MAGIC Collaboration), Science, 2008, 5884: 1752

[164] Y. Becherini et al. (H.E.S.S. Collaboration), AIP Conf. Proc., 2012, 1505: 490

[165] F. Aharonian et al. (H.E.S.S. Collaboration), ApJL, 2009, 695: L40

[166] A.A. Abdo et al. (Fermi-LAT Collaboration) ApJ, 2010, 719: 1433

[167] F.M. Rieger, Mem. S.A.It. 2012, 83:127

[168] R-Z. Yang et al., A\&A, 2012, 542: A19

[169] F. Aharonian et al. (HEGRA Collaboration), A\&A, 2003, 403: L1
[170] F.M. Rieger and F. Aharonian, MPLA, 2012, 27: 12300301

[171] F. Aharonian et al. (H.E.S.S. Collaboration), Science, 2006, 314: 1424

[172] J. Albert et al. (MAGIC Collaboration), ApJ, 2008, 685: L23

[173] E. Aliu et al. (VERITAS Collaboration), ApJ, 2012, 746: 141

[174] A. Abramowksi et al., ApJ, 2012, 746: 151

[175] V.A. Acciari et al. (VERITAS, MAGIC, VLBA M87 and H.E.S.S. Collab.), Science, 2009, 325: 444

[176] J. Aleksic et al. (MAGIC Collaboration), ApJL, 2010, 723: 207

[177] T.A. Rector, J.T. Stocke and E.S. Perlman, ApJ, 1999, 516 145

[178] J. Aleksic et al. (MAGIC Collaboration), A\&A, 2012, 539: 2

[179] A.M. Brown and J. Adams, MNRAS, 2011, 413: 2785

[180] M. Kadler et al., A\&A, 2012, 538: L1

[181] F. Acero et al. (H.E.S.S. Collaboration), Science, 2009, 326: 1080

[182] A. Abramowksi et al. (H.E.S.S. Collaboration), ApJ, 2012, 757: 158

[183] V.A. Acciari et al. (VERITAS Collaboration), Nature, 2009, 462: 770

[184] A.R. Bell, APh, 2012, in press (doi: 10.1016/j.astropartphys.2012.05.022)

[185] P. Meszaros, AIP Conf. Proc., 2009, 1085: 38

[186] F. Aharonian et al. (H.E.S.S. Collaboration), A\&A, 2009, 495: 505

[187] V. Acciari et al. (VERITAS Collaboration), ApJ, 2011, 743: 62 .

[188] J. Albert et al. (MAGIC Collaboration), ApJ, 2007, 667: 358

[189] A. Ackermann et al. (Fermi-LAT Collaboration), ApJ, 2011, 729: 114

[190] P. Blasi, S. Gabici and G. Brunetti, IJMPA 2007, 22: 681

[191] A.M. Atoyan and H.J. Voelk, ApJ, 2000, 535: 45

[192] V. Petrosian, A. Bykov and Y. Raphaeli, Space Science Reviews, 2008, 134: 191

[193] G. Vannoni et al., A\&A, 2011, 536: A56

[194] S. Inoue, F.A. Aharonian and N. Sugiyama, ApJ, 2005, 628: L9

[195] S.R. Kelner and F.A. Aharonian, Phys. Rev. D, 2008, 78: 034013

[196] A. Pinzke, C. Pfrommer and L. Bergström, Phys. Rev. D, 2011, 84: 123509

[197] J. Aleksic et al. (MAGIC Collaboration), A\&A, 2012, 541: A99

[198] T. Arlen et al. (VERITAS Collaboration), ApJ, 2012, 757: 123 
[199] F. Aharonian et al. (H.E.S.S. Collaboration), A\&A, 2009, 502: 437

[200] A. Abramowski et al. (H.E.S.S. Collaboration), A\&A, 2012, in press (arXiv:1208.1370)

[201] A. Abramowski et al. (H.E.S.S. Collaboration), ApJ, 2012, 750: 123

[202] F.M. Rieger, IJMPD, 2011, 20: 1547

[203] G. Pedaletti, S.J. Wagner and F.M. Rieger, ApJ, 2011, 738: 142

[204] G.V. Kulikov and G. B. Kristiansen, J Exp Theor. Phys., 1958, 35: 441

[205] V. Hess, Physikalische Zeitschrift, 1912, 13:1084

[206] V.L. Ginzburg and S.I. Syrovatskii, The Origin of Cosmic Rays, New York: Macmillan, 1964

[207] T.K. Gaisser, Cosmic rays and particle physics, Cambridge University Press, 1990

[208] V.S. Berezinskii et al., Astrophysics of Cosmic Rays, Amsterdam 1990, edited by V.L. Ginzburg

[209] L.O' Drury, F.A. Aharonian and H.J. Völk, A\&A, 1994, 287: 959

[210] F.A. Aharonian, L.O. Drury and H.J. Völk, A\&A, 1994, 285: 654

[211] E.G. Berezhko and H. Völk, A\&A, 2008, 492: 695

[212] G. Morlino et al., MNRAS, 2009, 392: 240

[213] S. Gabici, F.A. Aharonian and S. Casanova, MNRAS, 2009, 396: 1629

[214] D.C. Ellison and A.M. Bykov, ApJ, 2011, 731: 87

[215] S. Casanova et al., PASJ, 2010, 62: 765

[216] F.A. Aharonian and A.M. Atoyan, A\&A, 1996, 309: 917

[217] E. Parizot et al., A\&A, 2004, 424: 747

[218] J.C. Higdon and R.E. Lingenfelter, ApJ, 2005, 628: 738

[219] A.A. Bykov et al., AIP Conf. Proc., 2012, 1505: 46

[220] A. Atoyan et al., ApJ, 2006, 642: L153

[221] O. Adriani et al., Nature, 2009, 458: 607

[222] O. Adriani et al., Science, 2011, 332: 69

[223] A. Neronov, D.V. Semikoz and A.M. Taylor, Phys. Rev. Lett., 2012, 108: 051105

[224] S. Gabici et al., SF2A, 2010, (arXiv:1009.5291)

[225] J.G. Kirk, Y. Lyubarsky and J. Petri, ASSL 2009, 357: 421 [226] J. Arons, ASSL 2009, 357: 373

[227] B. Bucciantini, Int. J. Mod. Phys. Conf. Ser. 2012, 8: 120 [228] S.V. Bogovalov and F.A. Aharonian, MNRAS, 2000: 313, 504

[229] L. Ball and J.G. Kirk, APh, 2000: 12, 335

[230] C.F. Kennel and F.V. Coroniti, ApJ, 1984: 283, 710

[231] V.S. Beskin and R.R. Rafikov, MNRAS, 2000: 313, 433

[232] M. Lemoine and G. Pelletier, AIP 2012: 1439, 194

[233] J. Petri and Y. Lyubarsky, A\&A 2007: 473, 683
[234] Y. Lyubarsky and M. Liverts, ApJ, 2008: 682, 1436

[235] L. Sironi and A. Spitkovsky, ApJ 2011: 741, 39

[236] I. Arka and J.G. Kirk, ApJ, 2012: 745, 108

[237] M.L. Lister et al., AJ, 2009: 138, 1874

[238] B.G. Piner et al., ApJ, 2012: 758, 84

[239] B.G. Piner et al., ApJ, 2010: 723, 1150

[240] M. Georganopoulos and D. Kazanas, ApJ, 2003, 599: L5

[241] A. Levinson, ApJ, 2007, 671: L29

[242] G. Ghisellini, F. Tavecchio and M. Chiaberge, A\&A, 2005, 432: 401

[243] F.M. Rieger and P. Duffy, ApJ, 2004, 617: 155

[244] J.C. McKinney, MNRAS, 2006, 368: 1561

[245] O. Porth and C. Fendt, ApJ, 2010, 709: 1100

[246] M. Livio, G.I. Ogilvie and J.E. Pringle, ApJ, 1999, 512 100

[247] J.W. Broderick and F.P Fender, MNRAS, 2011, 417: 184

[248] R. Daly, MNRAS, 2011, 414: 1253

[249] J. Aleksic et al. (MAGIC Collaboration), ApJL, 2011, 730: L8

[250] O. Bromberg and A. Levinson, ApJ, 2009, 699: 1274

[251] F. Tavecchio et al., A\&A, 2011, 534: 86

[252] C.D. Dermer, K. Murase and H. Takami, ApJ, 2012, 755: 14

[253] F. Taveccio et al. , Phys. Rev. D, 2012, 86: 085036

[254] K. Nalewajko et al., MNRAS, 2012, 425: 2519

[255] F. Tavecchio and G. Ghisellini, preprint 2012, arXiv: 1209.2291

[256] C.M. Gaskell, New Astronomy Review, 2009, 53: 140

[257] B. Czerny and K. Hryniewicz, A\&A, 2011, 525: L8

[258] T.G. Arshakian et al., MNRAS, 2010, 401: 1231

[259] P.S. Smith, G.D. Schmidt and B.T. Jannuzi, Fermi Symposium 2011, arXiv:1110.6040

[260] E.P. Farina et al., MNRAS, 2012, 424: 393

[261] D. Giannios, D.A. Uzdenksy and M.C. Begelman, MNRAS, 2010, 402: 1649

[262] Y-D. Cui et al., ApJ, 2012, 746: 177

[263] M.V. Barkov, V. Bosch-Ramon and F.A. Aharonian, ApJ, 2012, 755: 170

[264] A. Levinson and F. Rieger, ApJ, 2011, 730: 123

[265] A. Abramowski et al. (H.E.S.S. Collaboration), A\&A, 2010, 520: A83

[266] A.A. Abdo et al. (Fermi-LAT Collaboration), ApJ, 2010, 722: 520

[267] P. Uttley, I.M. McHardy and S. Vaughan, MNRAS, 2005 359: 345

[268] J. Biteau and B. Giebels, A\&A, 2012, in press (arXiv:1210.2045)

[269] G. Ghisellini and F. Tavecchio, MNRAS, 2008, 386: L28 
[270] D. Giannios, D.A. Uzdenksy and M.C. Begelman, MNRAS, 2009, 395: L29

[271] M. Barkov et al., ApJ, 2012, 749: 119

[272] J.G. Kirk and I. Mochol, ApJ, 2011, 729: 104

[273] R. Narayan and T. Piran, MNRAS, 2012, 420: 604

[274] M.C. Begelman, A.C. Fabian and M.J. Rees , MNRAS, 2008, 384: L19

[275] T. Boutelier, G. Henri and P-O. Petrucci, MNRAS, 2008, 390: 73

[276] L.M. Heil, S. Vaughan and P. Uttley, MNRAS, 2012, 422: 2620

[277] Y.E. Lyubarskii, MNRAS 1997, 292: 679

[278] F.M. Rieger and F. Volpe, A\&A, 2010, 520: 23

[279] V. Bosch-Ramon and D. Khangulyan, IJMPD, 2009, 18: 347

[280] G. Dubus, A\&A, 2006, 456: 801

[281] G. Dubus, B. Cerrutti and G. Henri, A\&A, 2010, 516: 18

[282] J. Moldon et al., ApJ, 2011, 732: L10

[283] M. Ribo et al., A\&A, 2008, 481: 17

[284] M. Massi, E. Ros, and L. Zimmermann, A\&A, 2012, 540: A142

[285] J. Moldon, M. Ribo and J. Paredes, AIP Conf Proc., 2012, 1505: 386 (arXiv: 1210.7702)

[286] M. Perucho, V. Bosch-Ramon and D. Khangulyan, A\&A, 2010, 512: L4

[287] V. Bosch-Ramon and D. Khangulyan, PASJ, 2011, 63: 1023
[288] G.E. Romero et al., A\&A, 2007, 474, 15

[289] T. Takahashi et al., ApJ, 2009, 697: 592

[290] A. Abramowski et al. (H.E.S.S. Collaboration), A\&A, 2013, in press (arXiv:1301.3930

[291] V. Zabalza et al., A\&A, 2013 to appear (arXiv:1212.3222

[292] J. Takata et al., ApJ, 2012, 750: 70

[293] S.V. Bogovalov et al., MNRAS, 2012, 419: 3426

[294] M. Perucho and V. Bosch-Ramon, A\&A, 2012, 539: 57

[295] P. Bordas et al., A\&A, 2009, 497: 325

[296] H. Gast et al. (H.E.S.S. Collaboration), Proc. 32nd ICRC, Beijing 2011 (arXiv:1204.5860)

[297] http://www.mpi-hd.mpg.de/hfm/HESS/pages/home/som/ 2011/01/

[298] A.A. Abdo et al. (Fermi-LAT Collaboration), ApJ, 2009, 701: L123

[299] A.A. Abdo et al. (Fermi-LAT Collaboration), ApJ, 2009, 706: L56

[300] A. Abramowski et al. (H.E.S.S. Collaboration), PRL, 2013,

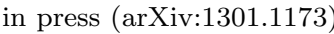

[301] D.I. Jones et al., ApJL, 2012, 747: L12

[302] Q. Yuan, S. Liu and X. Bi, ApJ, 2012, 761: 133

[303] S. Sahu, B. Zhang and N. Fraija, Phys Rev D, 2012, 85: 043012

[304] K. Mannheim, D. Elsässer and O. Tibolla, 2012, APh, 35: 797

[305] S. Ohm and J. Hinton, 2013, MNRAS, 429: L70 